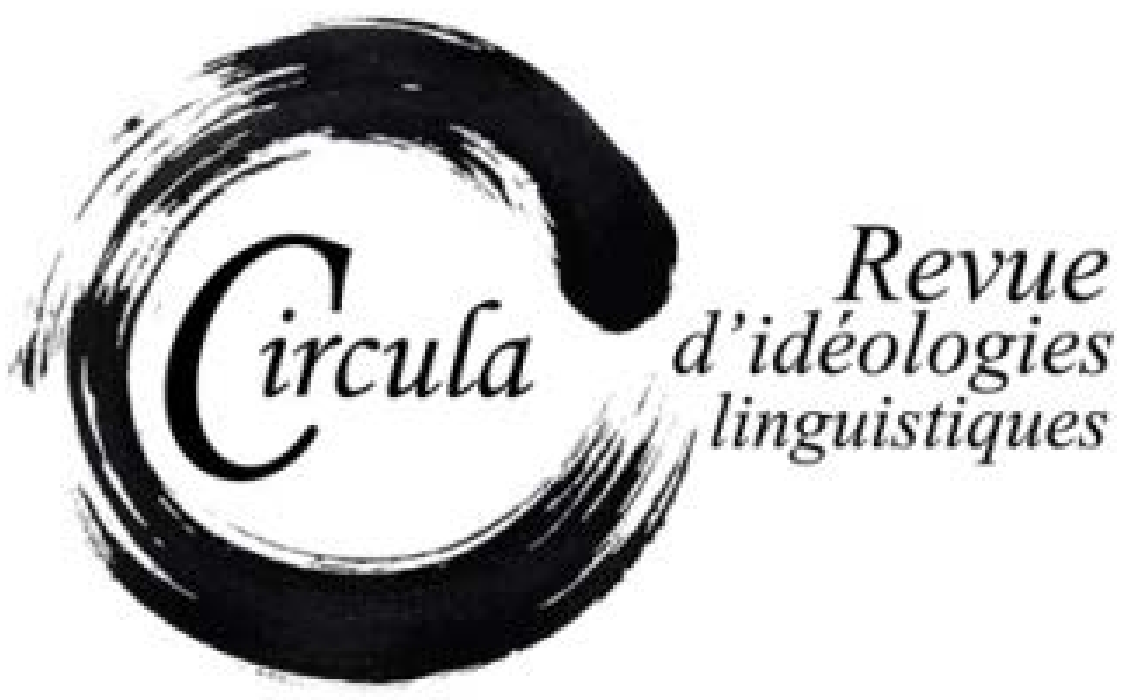

TITRE: REPRÉSENTATIONS DES LANGUES EN CONTEXTE PLURILINGUE ALGÉRIEN

Auteur(s): Zakaria Ali-Bencherif, Université de TlemCEn - CRASC Oran et AZZEddine MAHIEDDINE, UNIVERSITÉ DE TLEMCEN

Revue: CirCula, NUMÉRO 3

PAgES: $163-196$

ISSN: 2369-6761

Directeur: Bruno Maurer, Université PAul-VAlÉry - MontPellier 3

URI: HTTP://HDL.HANDLE.NET/11143/9691

DOI: $10.17118 / 11143 / 9691$ 


\title{
Représentations des langues en contexte plurilingue algérien
}

\author{
Zakaria Ali-Bencherif, Université de Tlemcen - CRASC Oran \\ zakaria.alibencherif@gmail.com
}

Azzeddine Mahieddine, Université de Tlemcen azmahieddine@yahoo.fr

Résumé : Le début des années 2000 a constitué un tournant dans l'évolution de la situation sociolinguistique de l'Algérie, marqué notamment par une ouverture sur le plurilinguisme qui se manifeste aujourd'hui sur le terrain comme un fait établi. Cette dynamique appelle un regard renouvelé sur les représentations qu'ont les locuteurs algériens des différentes langues en contact. C'est l'objectif de cet article qui présente les résultats d'une enquête sur les représentations sociales des quatre langues en présence (arabe standard, arabe dialectal, berbère et français) chez une population d'étudiants universitaires. Cette recherche, qui se base sur la méthode d'analyse combinée (Maurer, 2013) a permis de mettre au jour la structure de la représentation sociale de chaque langue et de voir la prégnance des images qui circulent chez les locuteurs.

Mots-clés : représentations sociales ; plurilinguisme ; action glottopolitique ; dynamique sociolinguistique et identitaire

\begin{abstract}
The beginning of the 2000s constituted a turning point in the evolution of the sociolinguistic situation of Algeria, characterized particularly by an opening on multilingualism. This dynamics requires a new vision of the representations the Algerian speakers have of their various languages in contact. The objective of this article is to present the results of a survey on the social representations of four languages in presence (standard arabic, dialectal, berber and french arabic) to a population of university students. This research, which bases itself on the combined method of analysis (Maurer, 2013), allowed to shed light on the structure of the social representation of every language and to see the dominance of the images which circulate among the speakers.
\end{abstract}

Keywords: social representations; multilingualism; glottopolitique action; sociolinguistics and identity dynamics 


\section{Introduction}

Interroger les représentations des langues en Algérie s'avère une question complexe du fait de la complexité de la situation (socio) linguistique et de sa dynamique. Plurilingue par excellence, l'Algérie a connu tout au long de son histoire des mutations (socio)linguistiques liées à des conjonctures historiques particulières. En tant qu'espace de confluences, ce pays doit en effet sa diversité à la rencontre et la cohabitation de peuples de langues et de cultures différentes qui lui ont donné une configuration linguistique où coexistent principalement l'arabe dialectal' ${ }^{1}$, l'arabe standard², le berbère ${ }^{3}$ et le français. Aujourd'hui, cette diversité linguistique est assujettie à une réalité imposée par la globalisation et l'ouverture sur le monde. Dans le contexte actuel, où le plurilinguisme n'est plus une exception, les représentations langagières sont marquées, à notre sens, par une dynamique tributaire du rôle que jouent les langues dans la vie quotidienne des individus et du poids de ces langues à l'échelle mondiale (Gasquet-Cyrus et Petitjean, 2009 ; Calvet et Calvet, 2013).

Cette dynamique que connait la société algérienne au niveau sociolinguistique appelle à une actualisation de la recherche sur les représentations. D’ailleurs, si en Algérie les recherches portant sur la description de la situation sociolinguistique et l'analyse des pratiques langagières abondent, celles qui traitent des représentations linguistiques restent encore peu nombreuses ${ }^{4}$. Ces dernières utilisent généralement des méthodes d'observation et d'analyse classiques (questionnaires et entretiens).

Dans la présente contribution, nous avons souhaité apporter un regard renouvelé sur les représentations que se font actuellement les locuteurs algériens des principales langues en contact en Algérie. Cette question sera abordée à la lumière d'une nouvelle méthodologie d'enquête : la méthode d'analyse combinée (Maurer, 2013).

1. L'arabe dialectal (ou arabe algérien) est le vernaculaire majoritaire. Cet idiome, en l'absence d'une normalisation, reste limité à l'usage oral. Il existe des variantes de l'arabe dialectal algérien en fonction des régions.

2. L'arabe standard est la forme moderne de l'arabe, normalisé au $19^{e}$ siècle à partir de l'arabe " classique ». C'est la langue officielle des pays arabes et celle transmise par l'institution éducative.

3. Il existe en réalité plusieurs variétés de ce qu’on appelle communément le berbère : le kabyle, le chaoui, le mozabite, le tergui, etc. Se pose à ce propos un problème de dénomination (Cheriguen, 2007 ; Bektache, 2014).

4. Les recherches notables sur les pratiques langagières et les représentations des langues en Algérie remontent à la fin des années 80. Certaines ont traité de la question d'arabisation, des politiques linguistiques et des questions identitaires, et ont interrogé dans le même temps les représentations et attitudes des locuteurs envers les langues en présence (Grandguillaume, 1983 ; Morsly, 1988). D’autres, qui se sont davantage penchés sur les représentations des langues, ont focalisé sur le français (Morsly, 1990) et son apprentissage scolaire (Boudebia-Baala, 2012 ; Ait Hamou-Ali, 2004). Enfin, certaines se sont intéressées aux incidences des représentations sur la réussite de la politique linguistique (Grine, 2009; Arezki, 2010). 


\section{Situation sociolinguistique en Algérie : des mutations à l'œuvre}

Complexe, la situation linguistique de l'Algérie suscite régulièrement des débats passionnés voire enflammés. La compréhension des mutations, du rapport qu'entretiennent les Algériens avec leurs langues, et des tensions que connaissent les débats sur les langues nécessite un petit regard rétrospectif.

Au lendemain de l'indépendance politique de l'Algérie (1962), et après 132 ans de colonisation française, l'État algérien a aussitôt lancé une politique d'arabisation, conçue comme une entreprise de récupération identitaire et de parachèvement de l'indépendance au niveau culturel. L'arabe standard, est ainsi promu au rang de seule langue nationale et officielle et devient le symbole de l'identité et la souveraineté nationales.

Le français, largement perçu à l'époque comme langue du colonisateur, et stigmatisé dans le discours du pouvoir, est toutefois maintenu comme «langue étrangère », en attendant le parachèvement de l'arabisation qui devait transformer le visage linguistique de l'Algérie au profit de l'arabe standard. Il n'en fut rien. Le français continue à être très présent dans la société algérienne et semble même connaitre depuis plus d'une décennie un regain de vitalité dans certains secteurs (Sebaa, 2002). Par ailleurs, l'édification du nouvel État-nation, inscrite dans la sphère de l'arabité/islamité, se fit également au détriment des langues maternelles (arabe dialectal et berbère) auxquelles on refusa tout statut et qui furent bannies de la sphère de l'officialité. En d'autres termes, la politique linguistique d'unilinguisme tourna le dos à la diversité linguistique et culturelle du pays (langues parlées, berbérité) susceptible de menacer l'unité nationale, et ce malgré les résistances affichées notamment par les berbérophones, durant plusieurs décennies (Zenati, 2004).

Cet état de fait a entrainé des conflits diglossiques latents et patents et dont les conséquences se voyaient clairement à travers un discours épilinguistique de rejet, de haine de soi (Benrabah, 1999).

Sur le terrain, les écarts se creusent entre la réalité sociolangagière et les décisions prises à l'égard des langues en présence. À ce propos, Khaoula Taleb-Ibrahimi (1997) faisait remarquer l'existence d'un hiatus entre politique linguistique, pratiques langagières et représentations qu'elle replace dans une vue d'ensemble de la situation linguistique de l'Algérie.

Concernant les résultats de l'arabisation, ils sont loin d'être à la mesure du programme. En effet, si l'on enregistre des avancés remarquables dans certains secteurs (l'éducation et la justice notamment), le français continue à occuper une place importante voire privilégiée dans plusieurs domaines de premier plan (enseignement supérieur, santé, sciences et techniques, économie, médias...). Le statut de cette langue demeure cependant ambigu, comme le décrit Rabah Sebaa (2002) : 
Sans être la langue officielle, la langue française véhicule l'officialité, sans être la langue d'enseignement, elle reste une langue de transmission du savoir, sans être la langue identitaire, elle continue à façonner l'imaginaire culturel collectif de différentes formes et par différents canaux. Et sans être la langue d'université, elle est la langue de l'université. (Sebaa, 2002 : 85)

Cette ambivalence face à une langue longtemps stigmatisée traduit « les paradoxes de la domination linguistique » (Colonna, 2013).

Par ailleurs, les berbérophones, qui ont toujours été réfractaires à la politique linguistique d'arabisation, ont constamment manifesté contre l'exclusion du berbère, jusqu'à sa reconnaissance comme seconde langue nationale en 2002 (Zenati, 2004).

En revanche, il n'y a jamais eu de revendications pour la promotion de l'arabe dialectal comme langue nationale ou officielle. Ce qui s'explique notamment par l'existence d'une ambigüité dans le rapport à l'arabe dialectal (Benrabah, 1993, 1999). Il n'est pas rare en effet que ce vernaculaire majoritaire ne soit pas considéré, par le commun des locuteurs, comme une langue à part entière mais comme une version déformée de l'arabe standard. Ce dernier est alors souvent considéré comme la langue maternelle, bien qu'il soit absent de la communication orale quotidienne. Cette vision fait que, du point de vue de la masse parlante, il n'y a pas de situation de conflit ou d'oppression entre l'arabe standard et l'arabe dialectal (contrairement au berbère). Ainsi, l'arabe dialectal reste en quelque sorte à l'ombre de l'arabe standard (Chachou, 2013b).

Les événements d'octobre 1988 tout comme la décennie noire (années 90) constituent des tournants importants dans l'histoire de l'Algérie moderne. Cela a conduit, après plusieurs années de chao, à des changements profonds sur les plans politique, économique et culturel. Le début des années 2000 fut marqué par un retour de l'Algérie sur la scène mondiale et par l'ouverture de plusieurs chantiers de réformes dont le secteur l'éducation. Cette période, constitue également un tournant dans l'évolution de la situation sociolinguistique de l'Algérie. Plusieurs indicateurs vont dans ce sens.

Le changement d’attitude du président de la république Abdelaziz Bouteflika, élu en 1999, vis-à-vis de la politique linguistique menée jusque-là est clairement affiché, comme le montre la déclaration suivante, faite devant l'assemblée nationale à Paris, le 16 juin 2000 :

La langue française et la haute culture qu'elle véhicule restent pour l'Algérie, des acquis importants et précieux que la réhabilitation de l'arabe, notre langue nationale, ne saurait frapper d'ostracisme. C'est là une richesse à même de féconder notre propre culture et c'est pourquoi le français, à l'instar d'autres langues modernes, et plus encore en raison de ses vertus intrinsèques et de son ancienneté dans notre pays, gardera une place qu'aucun complexe, aucun ressentiment ni aucune conjoncture quelconque ne saurait lui disputer. (Le Monde, 17 juin $2000: 18)$ 
La langue française continue, comme l'affirme Khaoula Taleb-Ibrahimi (2004 : 216) « [à jouir] d'une position prééminente dans le marché linguistique par sa prépondérance dans le monde du travail et de l'économie ». D'ailleurs, la réforme du système éducatif, lancée en 2000, lui a accordé une place privilégiée. Une première décision avait fixé le début de son enseignement à la deuxième année primaire. Mais rapidement, les contraintes du terrain repoussèrent son introduction à la troisième année primaire (rentrée 2006-2007). Quoi qu'il en soit, la revalorisation des langues étrangères et l'ouverture sur la pluralité linguistique est nettement affichée dans les finalités de l'enseignement:

Le monolinguisme ne peut contribuer au développement du pays. II ne permet ni l'ouverture sur le monde, ni l'accès aux savoirs et aux connaissances scientifiques élaborées ailleurs, empêchant ainsi l'établissement d'un dialogue fécond avec les autres cultures et civilisations. (Bulletin officiel de l'éducation nationale, 2008 : 17)

Par ailleurs, les questions linguistique et identitaire, notamment à l'égard de la langue et la culture berbères, sont revenues à l'ordre du jour. II a fallut attendre les événements du printemps noir en 2001 pour que le berbère soit reconnu en 2002 comme seconde langue nationale à côté de l'arabe standard. Cette reconnaissance marque un dépassement du modèle jacobin ${ }^{5}$ de politique linguistique et une ouverture vers un plurilinguisme institutionnalisé voire une unité nationale basée sur le plurilinguisme (Taleb-Ibrahimi, 1995) et les valeurs sociales des Algériens. L'enseignement ${ }^{6} \mathrm{du}$ berbère se développe ${ }^{7}$, essentiellement dans les régions berbérophones qui restent toutefois minoritaires d'un point de vue démolinguistique. Dès lors, affirme Khaoula Taleb-Ibrahimi (2004: 211), «[i]l faut donner à cette langue ou du moins à ces variantes régionales, les moyens nécessaires pour qu'elle(s) puisse(nt) occuper pleinement sa (leurs) place(s) dans le paysage linguistique et culturel du pays ». Par ailleurs, des chaines de radio et de télévision berbérophones font leur apparition.

5. «Correspond au modèle jacobin toute politique linguistique centralisée qui confère à une seule langue le bénéfice d'être celle de l'État, à l'exclusion de toute autre. Ce qui implique que l'État n'utilise et ne propage que sa langue, et décourage, dissuade et entrave tout usage public d'autres parlers autochtones, quitte à n'en même pas reconnaitre l'existence autrement que dans la sphère des usages privés » (Breton Roland, 1999: 82).

6. L'enseignement du berbère, déjà lancé en 1995, se développe avec le soutien du ministère de l'éducation nationale, du Haut Commissariat à l'Amazighité (HCA), et du Centre National Pédagogique et Linguistique pour l'Enseignement de Tamazigh (CNPLET). Cela constitue une avancée remarquable qui a rendu visible cette langue jusque-là réprimée.

7. Notons qu'un colloque a été co-organisé en 2006 par le Ministère de l'éducation nationale et le CNPLET autour du thème «Tamazight langue nationale en Algérie : état des lieux et politique d’aménagement », en présence de plusieurs ministres, de représentants du Haut Conseil à la Langue Arabe, du secrétaire général du Haut Commissariat à l'Amazighité ainsi que des chercheurs spécialistes de la question. 
De son côté, l'arabe dialectal, même s'il ne bénéficie d'aucune reconnaissance officielle, connait un regain de vitalité. Son usage oral ${ }^{8}$ s'étend à la sphère médiatique avec l'ouverture de nombreuses chaines de télévision privées. De plus, il s'impose sous une forme écrite dans le paysage linguistique ${ }^{9}$ (essentiellement dans les affiches publicitaires et les enseignes de commerce), même s'il n'a toujours pas fait l'objet d'une normalisation. Certaines études (Abbes-Kara, 2011 ; Boukra, 2012) préconisent un enseignement des (en) langues maternelles pour les deux premières années de l'école afin d'éviter la rupture avec la réalité vécue (la socialisation langagière) que l'arabe standard ne traduit pas.

De l'avis de plusieurs chercheurs, les langues maternelles, en tant que « véhicules de la modernité » (Berrabah, 1993) et symboles de la citoyenneté (Elimam, 2004) devraient, à côté de l'arabe standard, assurer l'unité nationale et figurer explicitement comme composantes essentielles de l'identité algérienne. En effet, celle-ci, telle que mise en discours (Amrane, 2010), ne repose pas uniquement sur l'arabe standard comme le souhaitaient les décideurs au lendemain de l'indépendance, mais également sur d'autres valeurs infranationales (l'appartenance à un groupe ou une ethnie, une région, une classe sociale, etc.). En tout état de cause, la pluralité est aujourd'hui de mise... et s'avère la solution.

La dynamique sociolinguistique et l'action glottopolitique à l'œuvre font que le plurilinguisme prend de plus en plus d'ampleur et se manifeste sur le terrain comme un fait établi. Cela apparait clairement dans les différentes sphères de socialisation comme l'affichage, les médias, les pratiques langagières familiales (Sini, 2013) et les échanges ordinaires.

Finalement, plus d'un demi siècle après l'indépendance de l'Algérie, quelles images les locuteurs algériens, généralement plurilingues, ont-ils de ces différentes langues en contact? Traduisent-elles la dynamique sociolinguistique et identitaire? Dans quelle mesure ces représentations sont en lien avec le discours idéologique? Nous supposons que, malgré les écarts qui continuent d'exister entre décisions politiques, pratiques langagières et représentations, les images qu'attribuent les locuteurs algériens aux langues en présence traduisent, d'un côté, la réalité vécue, dite et interagie, et d'un autre côté, la dynamique (socio)linguistique, identitaire et glottopolitique.

8. L'arabe dialectal est très souvent utilisé en alternance avec le français, ce qui aboutit parfois à des formes métissées (Quéffelec et al., 2002).

9. Selon Richard Bourhis et Rodrigue Landry (2002), « [l]e paysage linguistique est constitué de la langue de la signalisation routière, de la toponymie, de l'affichage sur les bâtiments administratifs (écoles, hôpitaux, cours de justice, ministères) et de l'affichage commercial». 


\section{Méthodologie : population, outil et terrain}

Nous ne reviendrons pas ici sur une description de la méthode d'analyse combinée (Maurer, 2011, 2013) utilisée pour cette enquête, puisqu'elle fait déjà l'objet d'une présentation dans la contribution de Bruno Maurer ici-même.

La population d'enquête retenue pour cette étude est composée d'un total de 180 étudiants de l'université de Tlemcen, répartis sur trois filières différentes:

- 60 étudiants d'anglais (département d'anglais)

- 60 étudiants de français (département de français)

- 60 étudiants d'économie (école préparatoire en sciences économiques, commerciales et sciences de gestion)

Il s'agit d'étudiants de $1^{\mathrm{re}}, 2^{\mathrm{e}}$ et $3^{\mathrm{e}}$ année, de langues maternelles différentes (berbère et arabe algérien) et originaires de différentes régions d'Algérie.

Le nombre de questionnaires pour les quatre langues étudiées est de 240 par filière, soit un total de 720 questionnaires traités.

Tableau 1 : Population d'enquête : échantillonnage par langue et par filière

\begin{tabular}{|c|c|c|c|c|}
\hline $\begin{array}{c}\text { Population } \\
\text { Langues }\end{array}$ & $\begin{array}{c}\text { Étudiants de } \\
\text { langue française }\end{array}$ & $\begin{array}{c}\text { Étudiants de } \\
\text { langue anglaise }\end{array}$ & $\begin{array}{c}\text { Étudiants } \\
\text { d'économie }\end{array}$ & $\begin{array}{c}\text { Nombre de } \\
\text { questionnaires }\end{array}$ \\
\hline Arabe standard & 60 & 60 & 60 & 180 \\
\hline Arabe algérien & 60 & 60 & 60 & 180 \\
\hline Français & 60 & 60 & 60 & 180 \\
\hline Berbère & 60 & 60 & 240 & 720 \\
\hline $\begin{array}{c}\text { Nombre de } \\
\text { questionnaires }\end{array}$ & 240 & 240 & 240 \\
\hline
\end{tabular}

Nous avons choisi des étudiants universitaires comme population d'enquête car nous estimons que cette catégorie sociale dispose a priori d'un plus haut degré de conscience linguistique ${ }^{10}$ et se montre particulièrement sensible à la question linguistique qui présente pour elle un enjeu de taille. Notre enquête s'est déroulée en trois temps, sur le terrain universitaire, entre avril et mai 2014.

10. Selon Annie Longatte (1999: 68), « le degré de conscience linguistique s'exprime dans la plus ou moins grande clairvoyance avec laquelle les jeunes perçoivent leur propre situation sociolinguistique, et influe fortement sur les différentes valeurs qu'ils assignent aux langues ». 


\subsection{La pré-enquête}

Une pré-enquête, par questionnaire écrit (v. annexes), a permis de recueillir pour chaque langue les items qui nous ont permis de construire les quatre questionnaires finaux. Pour cette première étape nous avons sollicité 80 étudiants. Ils devaient dire ce qu'évoque pour eux chacune des quatre langues en complétant librement et de manière concise les énoncés suivants: "Pour moi, l'arabe classique est... », « Pour moi, l'arabe dialectal est... », «Pour moi, le berbère est... », « Pour moi, le français est... ». Avec ces 80 répondants, la saturation a rapidement été atteinte et nous avons pu dégager une série de 15 items pour chaque langue.

\subsection{La construction des questionnaires finaux}

Les questionnaires finaux ont donc été construits à partir des résultats de la pré-enquête. Loin de l'idéologie souvent dictée par le chercheur, les réponses recueillies nous ont permis de proposer 15 items pour chacune des quatre langues (v. questionnaires en annexes). Pour écarter tout malentendu lié à la langue, les questionnaires finaux ont été rédigés en arabe et en français.

Les enquêtés devaient donc remplir en notre présence les questionnaires correspondant aux quatre langues (arabe standard, arabe dialectal, berbère et français) en classant les items de 1 (" pour moi, c'est vraiment ça ») à 15 (" pour moi ce n'est pas du tout ça »). Autrement dit, l'étudiant devait classer les 15 items de chaque langue en fonction de la représentation qu'il en a.

Cette consigne, ainsi que la présentation du questionnaire final, diffère dans une certaine mesure de la technique proposée par l'auteur de la méthode (Maurer, 2013). Elle en constitue une variante ${ }^{11}$.

11. Telle que présentée par Bruno Maurer (2013), la consigne aura été la suivante : a) noter +2 les trois propositions qui paraissent le mieux évoquer la langue étudiée, b) noter -2 les trois propositions qui paraissent le moins bien évoquer la langue étudiée, c) noter +1 les trois propositions qui paraissent assez bien évoquer la langue étudiée, d) noter -1 les trois propositions qui paraissent assez mal évoquer la langue étudiée, e) mettre la note 0 aux trois propositions restantes. Dans notre cas, il s'agira tout simplement de convertir les résultats du classement de 1 à 15 suivant cette notation : +2 pour les trois premiers items, +1 pour les trois autres qui suivent et ainsi de suite. 


\subsection{L'administration des questionnaires et le recueil des données}

Notre présence lors de l'administration des questionnaires était indispensable afin d'expliquer la consigne et de veiller à ce qu'ils soient remplis avec soin. Les données recueillies ont finalement été intégrés dans le logiciel conçu à cet effet ${ }^{12}$, qui nous a livré, suite à un traitement automatique, les résultats suivants:

- les principaux résultats en termes de score d'adhésion, d'indice de consensus et de distances significatives;

- le graphe adhésion-consensus-distance;

- le schéma en 4 couronnes : zone de centralité maximum - couronne centrale - périphérie incertaine - périphérie marginale

Ces résultats vont être présentés et discutés dans la section qui suit.

\section{Représentations sociales des quatre langues : analyse des données}

Nous allons présenter et analyser successivement les résultats relatifs à chacune des quatre langues, à partir des calculs statistiques réalisés par le logiciel. Dans cet article, nous ne prenons pas en considération, pour une raison d'espace, la variable filière.

12. Logiciel disponible sur le site http://linguiste.iutbeziers.fr/. V. Bruno Maurer (2013) pour une présentation du logiciel et des traitements mathématiques qu'il réalise. 


\subsection{Représentation sociale de l'arabe standard}

\subsubsection{Degré d'adhésion, indice de consensus et distance}

Tableau 2 : Scores pour le degré d'adhésion et l'indice de consensus

\begin{tabular}{|c|c|c|c|c|}
\hline \multicolumn{2}{|c|}{ Degré d'adhésion } & \multicolumn{2}{c|}{ Indice de consensus } \\
\hline Langue de la religion & 1,91 & Langue de la religion & 0,204 \\
\hline Langue de l'identité & 1,02 & Langue de la télévision & 0,102 \\
\hline Langue maternelle & 0,9 & Belle langue & 0,086 \\
\hline Grande langue & 0,51 & Langue de l'administration & 0,085 \\
\hline Langue de la lecture/écriture & 0,34 & Langue de prestige & 0,085 \\
\hline Langue riche & 0,31 & & Langue de l'identité & 0,084 \\
\hline Langue de l'école & 0,26 & & Grande langue & 0,083 \\
\hline Langue des origines & 0,04 & & Langue du savoir & 0,082 \\
\hline Langue de la littérature & $-0,22$ & & Langue de la lecture/écriture & 0,082 \\
\hline Langue du savoir & $-0,23$ & & Langue riche & 0,081 \\
\hline Belle langue & $-0,46$ & & Langue de la littérature & 0,079 \\
\hline Langue de prestige & $-0,83$ & & Langue difficile & 0,077 \\
\hline Langue difficile & $-0,85$ & & Langue de l'école & 0,075 \\
\hline Langue de l'administration & $-1,07$ & & Langue des origines & 0,073 \\
\hline Langue de la télévision & $-1,57$ & & Langue maternelle & 0,071 \\
\hline
\end{tabular}

Graphe de la représentation : Arabe standard

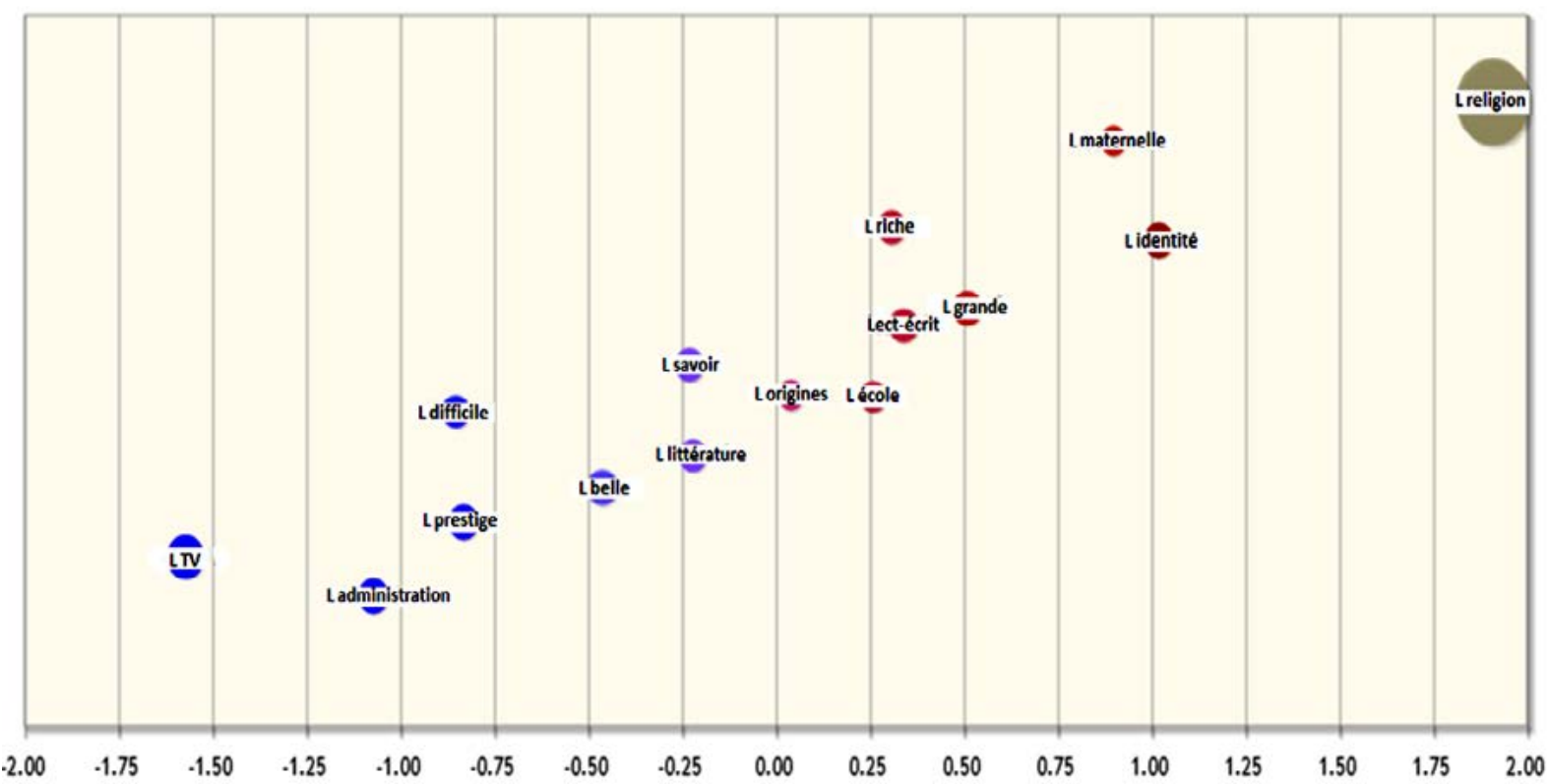

Graphe 1 : Graphe de la représentation sociale de l'arabe standard : adhésion - consensus 
Nous remarquons d'emblée le traitement particulier dont a fait l'objet l'item 6 (langue de la religion). Il est très fortement value à la fois au niveau de l'adhésion et du consensus et se détache très nettement des autres items. Le score d'adhésion $(1,91)$ est presque maximal.

L'item 9 (langue de l'identité) enregistre également une forte adhésion (1,02) ; il obtient toutefois un indice de consensus $(0,84)$ nettement inférieur à l'item 6 . L'item 14 (langue maternelle) arrive au troisième rang au niveau de l'adhésion, mais avec le plus faible indice de consensus. Les items qui obtiennent la plus faible adhésion sont les items 15 et 2 (langue de la télévision et langue de l'administration). Le fort rejet dont ils font l'objet est appuyé par des indices de consensus parmi les plus élevés.

Les autres items enregistrent des scores d'adhésion moyens, avec des indices de consensus plus ou moins significatifs $(>0,07)$. Ils sont rassemblés dans la couronne centrale (v. graphe infra).

Par ailleurs, le calcul des distances les plus faibles $(>1,40)$ montre que les couples d'items les plus fortement associés sont les items 2 (langue de l'administration) et 15 (langue de la télévision) - qui avaient également fait l'objet du plus fort rejet - et les items 7 (langue de l'école) et 11 (langue de la lecture/écriture).

\section{Schéma en couronnes de la structure de la représentation sociale de l'arabe standard}

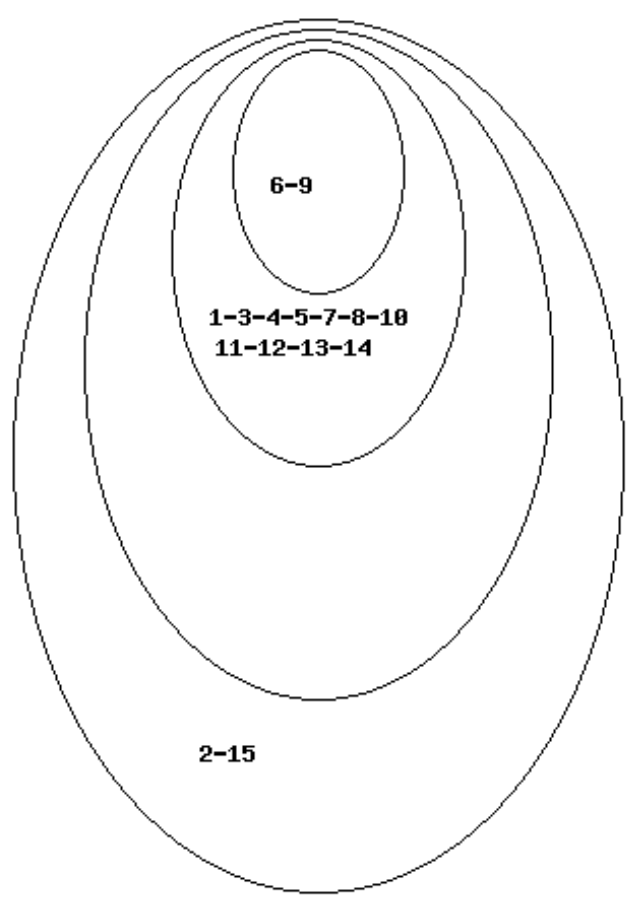

Graphe 2: Schéma en couronnes de la représentation sociale de l'arabe standard

\begin{tabular}{|c|c|}
\hline Item $\mathbf{1}$ & Langue du savoir \\
\hline Item $\mathbf{2}$ & Langue de l'administration \\
\hline Item $\mathbf{3}$ & Belle langue \\
\hline Item $\mathbf{4}$ & Langue difficile \\
\hline Item $\mathbf{5}$ & Langue des origines \\
\hline Item $\mathbf{6}$ & Langue de la religion \\
\hline Item $\mathbf{7}$ & Langue de l'école \\
\hline Item $\mathbf{8}$ & Langue de prestige \\
\hline Item $\mathbf{9}$ & Langue de l'identité \\
\hline Item $\mathbf{1 0}$ & Langue de la littérature \\
\hline Item $\mathbf{1 1}$ & Langue de la lecture/écriture \\
\hline Item $\mathbf{1 2}$ & Langue riche \\
\hline Item $\mathbf{1 3}$ & Grande langue \\
\hline Item $\mathbf{1 4}$ & Langue maternelle \\
\hline Item $\mathbf{1 5}$ & Langue de la télévision \\
\hline
\end{tabular}




\subsubsection{Commentaire}

La composition de la représentation sociale de l'arabe standard est dominée par les cognèmes «langue de la religion » et « langue de l'identité » qui occupent la zone de centralité maximum. Le premier, nous l'avons vu, est l'élément le plus prégnant.

Bien que l'arabe standard diffère dans une certaine mesure de l'arabe coranique, ce résultat montre que cette langue est, dans la représentation des locuteurs, indissolublement liée à l'islam. Ce qui rappelle les origines même de cette langue dans ce pays, qui a été, suite à la conquête arabo-musulmane, introduite avec la religion comme un tout a priori indissociable. Ce qui donne à cette langue une connotation religieuse voire un caractère sacré. Gilbert Grandguillaume (2010, n. p.) affirme que « la langue arabe classique entretient [...] un lien privilégié avec l'islam : emblème de l'identité musulmane elle est ressentie comme telle par les musulmans depuis des siècles ».

Ainsi, le noyau de la représentation, qui associe l'arabe standard à la « langue de la religion » et la « langue de l'identité », renferme des éléments fondamentaux de l'identité algérienne, profondément ancrés dans l'histoire de l'Algérie contemporaine. En effet, dans la première moitié du 20 siècle, la célèbre devise de Cheikh Ben Badis ${ }^{13}$, «l'Algérie est notre patrie, l'arabe est notre langue et l'islam est notre religion », reprise par les militants révolutionnaires, figurait comme un repère identitaire de l'Algérie colonisée. Au lendemain de l'indépendance, l'identité algérienne se définissait dans le discours idéologique comme ancrée exclusivement dans l'arabité et l'islamité. C'est ainsi que dans la Charte nationale de 1976, nous pouvons lire:

Le peuple algérien se rattache à la patrie arabe dont il est un élément indissociable. [...] se sont ajoutés progressivement à partir du $7^{\mathrm{e}}$ siècle, les autres éléments constitutifs de la nation algérienne, à savoir son unité culturelle, linguistique et spirituelle [...] l'islam et la culture arabe étaient un cadre à la fois universel et national [...]. Désormais, c'est dans ce double cadre [...] que va se déterminer le choix de notre peuple et se dérouler son évolution. (Charte nationale, 1976, titre premier, 83 ; cité par Djamel Zenati, 2004 : 139)

Remarquons que ce n'est qu'au tournant des années 2000 que ce discours réhabilitera la berbérité comme composante de l'identité algérienne.

La présence du cognème « langue de l'identité » dans le noyau de la représentation (conforté par le cognème « langue des origines » situé dans la couronne centrale) peut être interprétée comme une reprise de ce discours idéologique dominant. En effet, d'après nos résultats, c'est l'arabe standard plutôt que l'arabe dialectal ${ }^{14}$ qui est davantage considéré comme la référence identitaire alors même qu'il n'est pas la langue maternelle et demeure absent de la communication orale quotidienne.

13. Cheikh Ben Badis est le chef de file du mouvement réformiste qui se développa durant la première moitié du 20e siècle. Il créa l'association des Oulémas et prôna un nationalisme à caractère religieux.

14. L'arabe dialectal comme « langue de l'identité » a obtenu un score d'adhésion de -0,45. 
C'est dans le même ordre d'idée que nous pourrions interpréter le traitement de l'item 14 (langue maternelle) par nos enquêtés. En effet, s'il figure au troisième rang de l'adhésion, le plus faible indice de consensus qu'il obtient peut traduire une certaine confusion. Il se retrouve finalement dans la couronne centrale qui, par définition, rassemble les cognèmes les plus susceptibles d'évoluer. L'image de l'arabe standard comme langue maternelle va-t-elle, à l'avenir, être confirmée ou repoussée?

Les scores des items 15 (langue de la télévision) et 2 (langue de l'administration), qui ont fait l'objet d'un fort rejet, appuyé par un consensus très significatif, sont en lien avec la situation sociolinguistique du pays. Avec l'ouverture du champ audiovisuel et la multiplication des chaines de télévision privées, l'arabe dialectal et le berbère occupent une place de plus en plus importante voire prépondérante. De plus, les chaines satellitaires, notamment francophones, sont très prisées par les Algériens. Si bien que l'image de l'arabe standard comme langue de la télévision n'est plus de mise.

Par ailleurs, malgré les dispositions juridiques pour l'arabisation de l'administration, celle-ci continue à fonctionner en grande partie en français. De plus, la communication orale dans cette sphère sociale ne s'effectue généralement pas en arabe standard. Voilà ce qui explique sans doute le rejet de l'item «langue de l'administration».

Les autres cognèmes de la représentation sociale de l'arabe standard ne sont pas marqués. Certains vont dans le sens d'une idéalisation de cette langue ("grande langue », «langue riche »); d'autres font plus référence à l'écrit (« langue de lecture/écriture », « langue de la littérature»). 


\subsection{Représentation sociale de l'arabe dialectal}

\subsubsection{Degré d'adhésion, indice de consensus et distance}

Tableau 2: Scores pour le degré d'adhésion et l'indice de consensus

\begin{tabular}{|c|c|c|c|}
\hline \multicolumn{2}{|c|}{ Degré d'adhésion } & \multicolumn{2}{|c|}{ Indice de consensus } \\
\hline Dialecte & 1,02 & Langue inutile & 0,104 \\
\hline Langue du quotidien & 1 & Langue des analphabètes & 0,095 \\
\hline Facilite la communication & 0,88 & Langue la plus utilisée & 0,091 \\
\hline Langue la plus utilisée & 0,86 & Langue facile & 0,089 \\
\hline Langue du peuple & 0,76 & Langue du quotidien & 0,088 \\
\hline Langue facile & 0,23 & Dialecte & 0,088 \\
\hline Langue maternelle & 0,07 & Facilite la communication & 0,087 \\
\hline Langue métissée & 0,02 & Langue du peuple & 0,084 \\
\hline Être à l'aise & $-0,01$ & Langue dominante & 0,084 \\
\hline Langue de la rue & $-0,06$ & Langue métissée & 0,082 \\
\hline Langue de l'identité & $-0,45$ & Être à l'aise & 0,079 \\
\hline Langue dominante & $-0,46$ & Langue de la rue & 0,071 \\
\hline Non langue & $-0,84$ & Langue de l'identité & 0,071 \\
\hline Langue des analphabètes & $-1,41$ & Non langue & 0,07 \\
\hline Langue inutile & $-1,57$ & Langue maternelle & 0,059 \\
\hline
\end{tabular}

Graphe de la représentation : Arabe dialectal

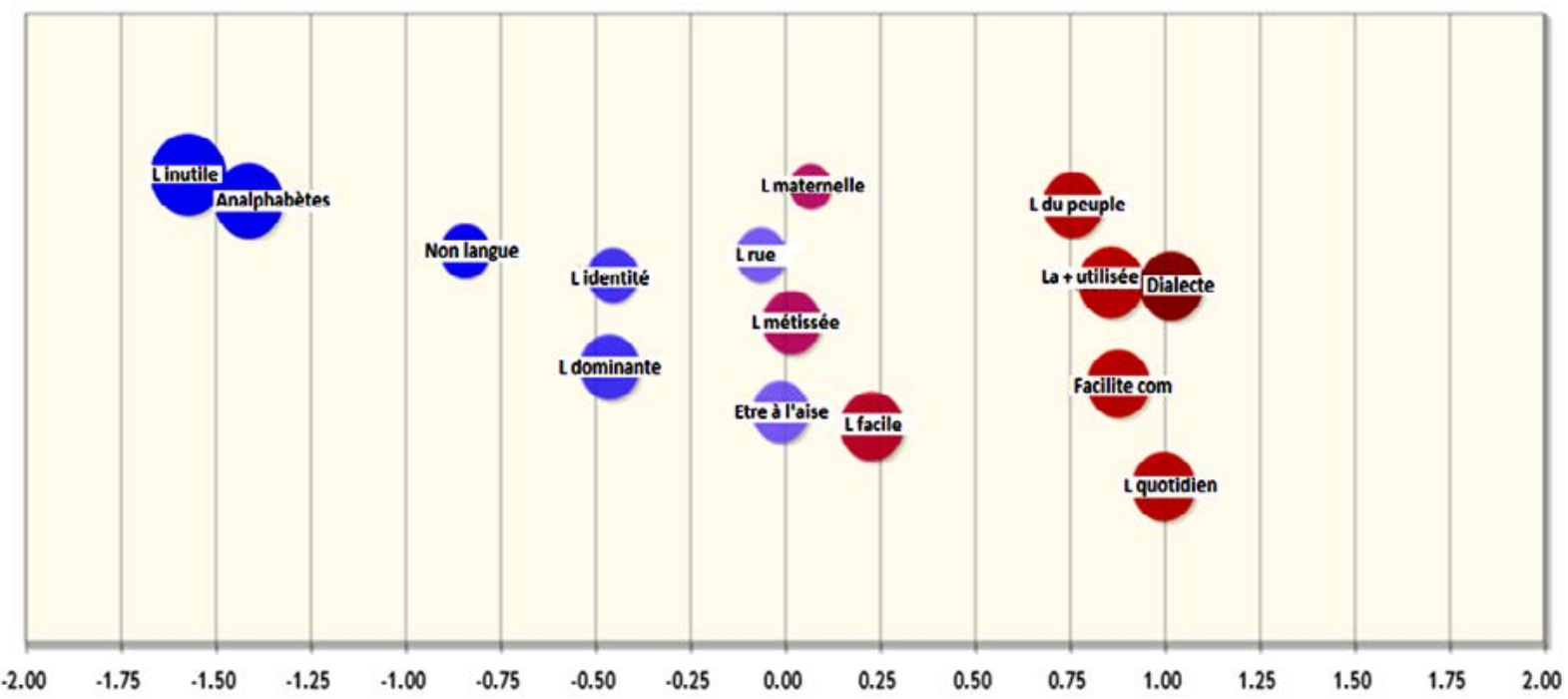

Graphe 3: Graphe de la représentation sociale de l'arabe dialectal : adhésion - consensus 
Les éléments qui font l'objet de la plus forte adhésion sont l'item 9 (dialecte) suivi de l'item 5 (langue du quotidien); ils reçoivent des indices de consensus identiques et assez significatifs.

À l'opposé, les items 2 (langue inutile) et 13 (langue des analphabètes) enregistrent la plus faible adhésion, appuyée par les scores de consensus les plus élevés; ce qui les repousse vers la périphérie extrême de la représentation.

Les items 6 (facilite la communication), 15 (langue la plus utilisée) et 3 (langue du peuple) reçoivent un degré d'adhésion inférieur à de l'item 9, mais ils enregistrent des indices de consensus significatifs.

Par ailleurs, le calcul des distances permet de dégager trois couples d'items fortement associés $(<$ à 1,4):

- 2 (langue inutile) et 13 (langue des analphabètes);

- 5 (langue du quotidien) et 15 (langue la plus utilisée);

- 6 (facilite la communication) et 15 (langue la plus utilisée);

Les associations par couples font ressortir le caractère utilitaire de l'arabe dialectal en tant que « dialecte » largement employé par les locuteurs (item15). Ceci renvoie aussi à sa première fonction, celle de langue de la communication quotidienne (5 et 6 ). Le caractère utilitaire ainsi que la fonction de langue vernaculaire qui lui est attribuée sont confirmés par le rejet de l'item 2 (langue inutile) qui est le plus faiblement valué $(-1,57)$, avec un traitement consensuel $(+0,104)$. Il en est de même pour l'item 13 (langue des analphabètes) qui se trouve dans la périphérie marginale avec une adhésion minimale $(-1,41)$ et un fort degré de consensus $(0,095)$. Mis en rapport avec l'item 2 , il permet de conclure de la valeur instrumentale que associée l'arabe dialectal. Si par ailleurs, le cognème 2 (langue inutile) enregistre un degré de consensus élevé, c'est surtout par comparaison à d'autres sphères d'activité (monde du travail, études, écrit, etc.). Le fort degré de consensus pour le rejet de l'item 13 (langue des analphabètes) témoigne sans doute de la valeur positive attribuée par le groupe à l'arabe dialectal en tant que langue indispensable pour la communication quotidienne. Cette langue, malgré l'absence d'un statut officiel, connaît sur le terrain une vitalité et une forte dynamique notamment par sa forte présence dans les médias (à l'oral), la publicité (Chachou, 2013a) et l'affichage urbain. Ceci pourrait être lu, à l'aune de certaines images réductrices générées massivement par le discours des lettrés arabisants, comme une dynamique des représentations. Certes l'arabe dialectal est loin d'être une langue enseignée ou d'enseignement mais il recèle une valeur inestimable quant à son caractère inventif et à son usage massif dans plusieurs sphères de socialisation langagière. 
Schéma en couronnes de la structure de la représentation sociale de l'arabe dialectal

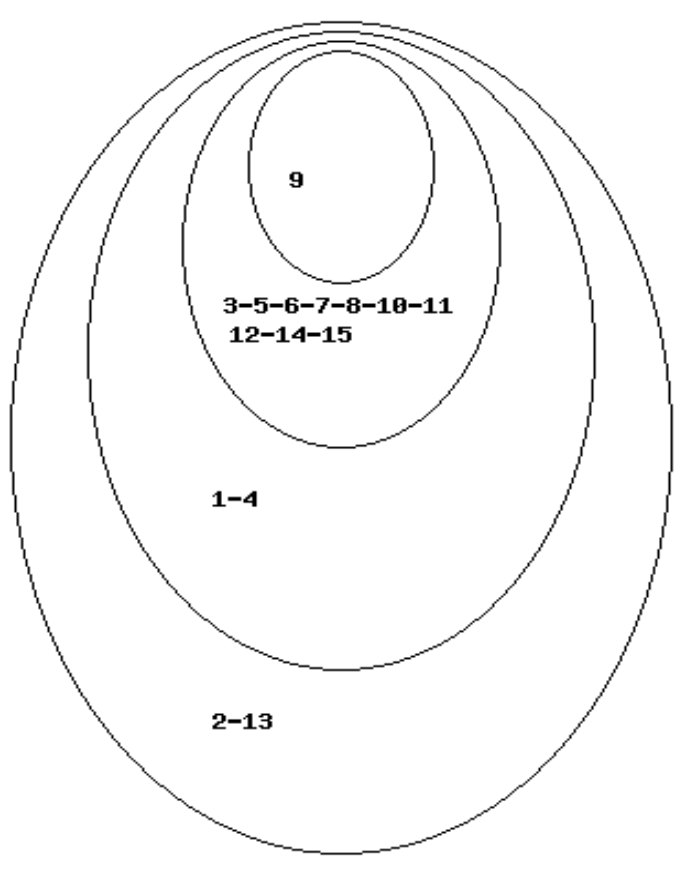

\begin{tabular}{|c|c|}
\hline Item $\mathbf{1}$ & Langue maternelle \\
\hline Item $\mathbf{2}$ & Langue inutile \\
\hline Item $\mathbf{3}$ & Langue du peuple \\
\hline Item $\mathbf{4}$ & Non langue \\
\hline Item $\mathbf{5}$ & Langue du quotidien \\
\hline Item $\mathbf{6}$ & Facilité de communication \\
\hline Item $\mathbf{7}$ & Langue de la rue \\
\hline Item $\mathbf{8}$ & Langue facile \\
\hline Item $\mathbf{9}$ & Dialecte \\
\hline Item $\mathbf{1 0}$ & Être à l'aise \\
\hline Item $\mathbf{1 1}$ & Langue de l'identité \\
\hline Item $\mathbf{1 2}$ & Langue métissée \\
\hline Item $\mathbf{1 3}$ & Langue des analphabètes \\
\hline Item $\mathbf{1 4}$ & Langue dominante \\
\hline Item $\mathbf{1 5}$ & Langue la plus utilisée \\
\hline
\end{tabular}

Graphe 4 : Schéma en couronnes de la représentation sociale de l’arabe dialectal

\subsubsection{Commentaire}

Le schéma en couronnes montre qu'un seul élément occupe la zone 1 de la centralité maximum ; il s'agit du cognème 9 (dialecte). Cette position montre bien la catégorisation faite à l'égard de l'arabe dialectal au sein de la communauté linguistique. À côté de cela, nous remarquons que le cognème 4 (non langue), qui obtient une adhésion moyenne, se retrouve dans la périphérie incertaine de la représentation. Ces deux items révèlent une catégorisation ${ }^{15}$ de l'arabe dialectal en tant que « dialecte » qui n'est en aucun cas perçu comme une « non langue ». La différence «langue » et « dialecte » ne semble pas poser, à notre sens, de problèmes en termes de cognitions, encore moins en termes de catégorisation du dialecte comme langue des analphabètes et de l'arabe standard comme langue des instruits. Toutefois, les cognèmes 9 et 13 constituent une polarisation significative à cet égard. Nous rappelons que d'un point de vu institutionnel l'arabe dialectal reste à l'ombre de l'arabe standard comme variété basse (langue inutile) mais indispensable et pratique en tant que vernaculaire ( darija ${ }^{16}$ ). Paradoxalement, l'attitude glottophage (Calvet, 1974) née du conflit diglossique incarné par la politique d'arabisation ne semble pas entraîner d'incidences sur les représentations sociales

15. V. Cyril Trimaille et Marinette Matthey (2013).

16. Le terme darija en arabe classique ou dardja en arabe dialectal renvoie à vernaculaire. 
de l'arabe dialectal puisque l'arabe standard n'a pas pris la place de l'arabe dialectal dans le quotidien de la société.

La zone 2 du schéma de la représentation regroupe un nombre important de cognèmes : 3, 5, 6, $7,8,10,11,12,14$ et 15. Ces cognitions sont relativement moyennes en termes d'adhésion mais reçoivent des indices de consensus significatifs. En effet, les deux cognèmes (6 et 15) ont fait l'objet d'une valuation importante. Leur concentration avec d'autres cognèmes dans la couronne centrale montre ostensiblement le rôle majeur de cette langue qui est fortement choisi par les membres du groupe. Ces cognèmes se combinent avec d'autres qui sont en lien avec les dimensions vernaculaire, identitaire et locale caractérisant cette langue : 3 (langue du peuple), 7 (langue de la rue), 8 (langue facile), 10 (être à l'aise), 11 (langue de l'identité), 12 (langue métissée), 14 (langue dominante). Nous constatons clairement à travers le graphe que ces éléments ne sont ni fortement choisis ni totalement repoussés. Par ailleurs, ce regroupement dans la zone centrale renseigne, à notre sens, sur la dynamique des représentations qui caractérise le rapport positif des répondants à l'égard de l'arabe dialectal ; cela s'explique également par le rejet de l'item 2 (langue inutile).

La zone de périphérie incertaine regroupe les items 1 (langue maternelle) et 4 (non langue), qui obtiennent des scores d'adhésion moyens mais avec des indices de consensus très bas, signe d'un désaccord important parmi les membres du groupe. On souligne ainsi une fluctuation quant à la perception de l'arabe dialectal en tant que langue maternelle.

Enfin, les cognèmes 2 et 13, fortement associés par la distance, se trouvent dans la périphérie marginale suite à un fort rejet, appuyé par les indices de consensus les plus élevés. 


\subsection{Représentation sociale du berbère}

\subsubsection{Degré d'adhésion, indice de consensus et distance}

Tableau 3 : Scores pour le degré d'adhésion et l'indice de consensus

\begin{tabular}{|c|c|c|c|}
\hline \multicolumn{2}{|c|}{ Degré d'adhésion } & \multicolumn{2}{|c|}{ Indice de consensus } \\
\hline Langue d'une minorité & 0,93 & Langue d'une minorité & 0,089 \\
\hline Langue du patrimoine & 0,73 & Langue de l'oralité & 0,088 \\
\hline Langue des origines & 0,68 & Langue des Maghrébins & 0,082 \\
\hline Symbole identité culturelle & 0,50 & Langue riche & 0,081 \\
\hline Langue secondaire & 0,29 & Langue secondaire & 0,079 \\
\hline Langue des Maghrébins & 0,01 & Belle langue & 0,079 \\
\hline Langue de l'oralité & 0,01 & Langue du patrimoine & 0,077 \\
\hline Langue menacée & $-0,02$ & Langue des origines & 0,075 \\
\hline Langue inutile & $-0,17$ & Symbole identité culturelle & 0,075 \\
\hline Langue de conflit & $-0,21$ & Langue étrangère & 0,075 \\
\hline Langue riche & $-0,43$ & Langue menacée & 0,073 \\
\hline Langue étrangère & $-0,46$ & Langue maternelle & 0,072 \\
\hline Belle langue & $-0,48$ & Langue de conflit & 0,070 \\
\hline Non langue & $-0,49$ & Non langue & 0,068 \\
\hline Langue maternelle & $-0,77$ & Langue inutile & 0,066 \\
\hline
\end{tabular}

Graphe de la représentation : Berbère

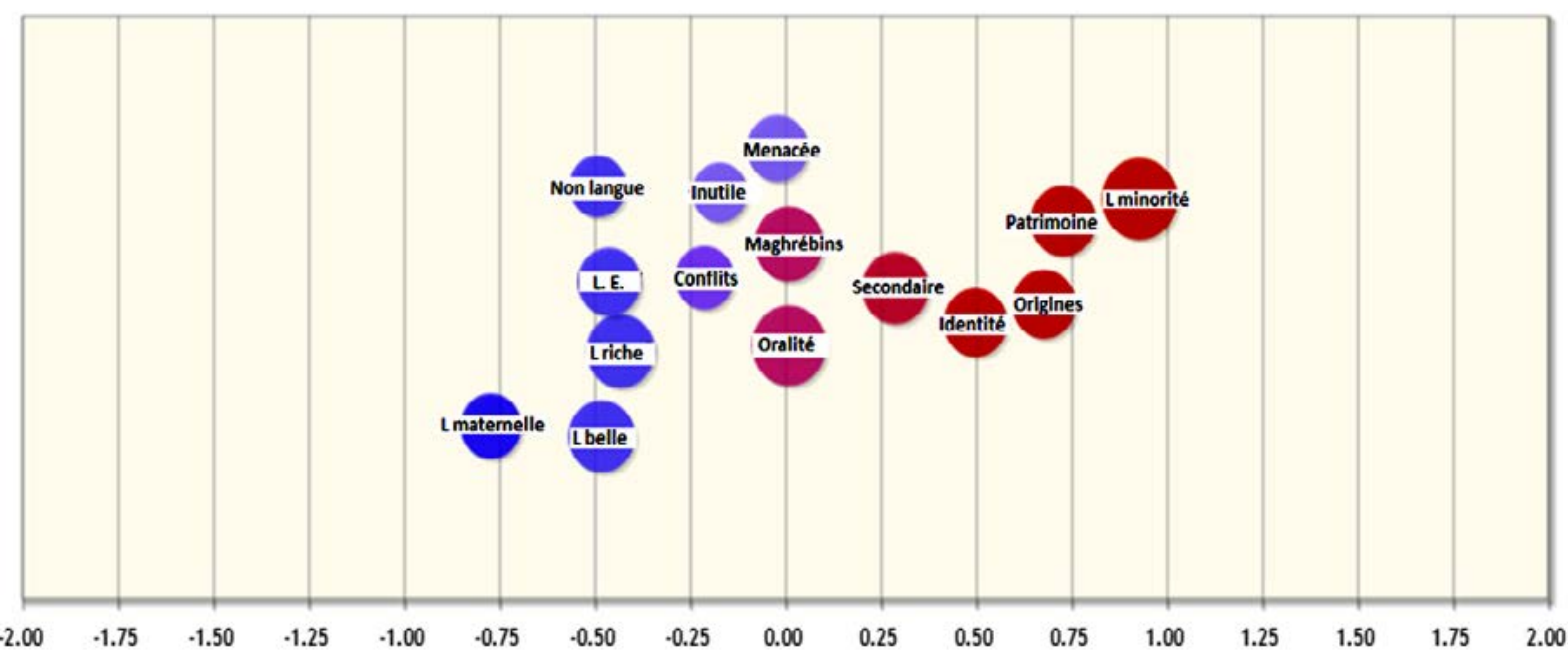

Graphe 5 : Graphe de la représentation sociale du berbère : adhésion - consensus 
Les données montrent qu'ils n'y a pas d'items fortement marqués du point de vue de l'adhésion/ rejet. L'indice de consensus, quant à lui, se limite à 0,089. Ce qui implique qu'aucun item ne figure dans le noyau central de la représentation ainsi que dans la périphérie marginale. Cela dit, ce sont les items 2 (langue d'une minorité) et 11 (langue du patrimoine) qui obtiennent les scores d'adhésion les plus élevés avec des indices de consensus significatifs.

Par ailleurs, les items 10 (non langue), 12 (langue inutile) et 5 (langue de conflit), qui sont valués négativement du point de vue de l'adhésion, sont marqués par un très faible consensus, témoignant d'un important désaccord dans leur traitement. Ce qui va les conduire à occuper la zone de périphérie incertaine de la représentation.

Les autres items enregistrent des scores d'adhésion non marqués avec des indices de consensus plus ou moins significatifs. Ils sont regroupés dans la couronne centrale.

Enfin le calcul des distances fait apparaître trois couples d'items qui sont les plus fortement associés:

- 8 (langue riche) et 9 (langue belle)

- 6 (symbole de l'identité culturelle) et 11 (langue du patrimoine)

- 1 (langue des origines) et 11 (langue du patrimoine) 


\section{Schéma en couronnes de la structure de la représentation sociale du berbère}

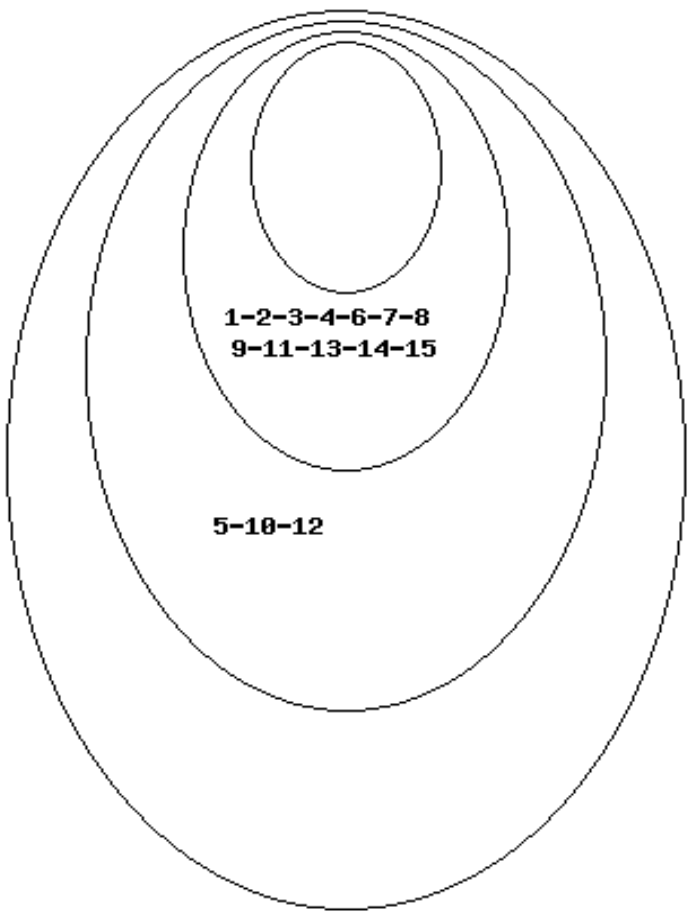

\begin{tabular}{|c|c|}
\hline Item $\mathbf{1}$ & Langue des origines \\
\hline Item $\mathbf{2}$ & Langue d'une minorité \\
\hline Item $\mathbf{3}$ & Langue secondaire \\
\hline Item $\mathbf{4}$ & Langue des Maghrébins \\
\hline Item $\mathbf{5}$ & Langue de conflit \\
\hline Item $\mathbf{6}$ & Symbole identité culturelle \\
\hline Item $\mathbf{7}$ & Langue étrangère \\
\hline Item $\mathbf{8}$ & Langue riche \\
\hline Item $\mathbf{9}$ & Belle langue \\
\hline Item $\mathbf{1 0}$ & Non langue \\
\hline Item $\mathbf{1 1}$ & Langue du patrimoine \\
\hline Item $\mathbf{1 2}$ & Langue inutile \\
\hline Item $\mathbf{1 3}$ & Langue menacée \\
\hline Item $\mathbf{1 4}$ & Langue de l'oralité \\
\hline Item $\mathbf{1 5}$ & Langue maternelle \\
\hline
\end{tabular}

Graphe 6 : Schéma en couronnes de la représentation sociale du berbère

\subsubsection{Commentaire}

Ces résultats sont à considérer relativement au fait que le groupe est constitué majoritairement de locuteurs arabophones (ayant pour langue maternelle l'arabe dialectal). Si l'on s'en tient au degré d'adhésion aux deux cognèmes 2 et 11, nous diront qu'ils renvoient plus à l'imaginaire collectif dont les valeurs sont partagées par la majorité des Algériens qu'ils soient berbérophones ou autre. Si le cognème (langue d'une minorité) reçoit un fort degré d'adhésion et un fort degré de consensus, c'est parce qu'il représente bien la réalité démolinguistique. II n'est pas surprenant de voir à l'autre extrémité du graphe, celle du rejet, le cognème 15 (langue maternelle) vu que le berbère n'est pas la langue maternelle des répondants. Ainsi, ces derniers lui attribuent le cognème de «langue du patrimoine » qui se trouve associé à « langue des origines » rapport fait avec sa dimension emblématique que le cognème 6 (symbole de l'identité culturelle) semble représenter. Les distances entre les cognèmes montrent bien cette association. Ceci nous permet de conclure, du point de vue de l'imaginaire linguistique (Anne-Marie Houdebine-Gravaud, 2002), que les répondants attribuent, par ce traitement consensuel, une image positive au berbère comme langue faisant partie de la réalité linguistique algérienne. 
Les cognèmes 2 (langue d'une minorité) et 11 (langue du patrimoine), qui ont fait l'objet d'une forte adhésion, se trouvent regroupés avec d'autres $(2,3,4,6,7,8,9,13,14,15)$ dans la couronne centrale. Cette concentration dans cette zone de la représentation renvoie à des opinions très répandues. Ceci nous amène à formuler l'hypothèse selon laquelle ces éléments seraient susceptibles d'une évolution dans le temps au vu des interventions réelles soulignées à l'égard du berbère qui est passé au rang de deuxième langue nationale. Le berbère est effectivement très présent dans les médias (publics et privés) et son enseignement se développe notamment dans les régions berbérophones.

Enfin, les trois cognèmes qui se trouvent regroupés dans la zone de périphérie incertaine (« langue de conflit », «non langue », « langue inutile »), traduisent les fluctuations attitudinales à l'égard du berbère ainsi que les discordances affichées entre les répondants. Ceci est sans doute du au fait que le berbère a longtemps été marginalisé par les hautes sphères de décisions. 


\subsection{Représentation sociale de la langue française}

\subsubsection{Degré d'adhésion, indice de consensus et distance}

Tableau 4 : Scores pour le degré d'adhésion et l'indice de consensus

\begin{tabular}{|c|c|c|c|}
\hline \multicolumn{2}{|l|}{ Degré d'adhésion } & \multicolumn{2}{|l|}{ Indice de consensus } \\
\hline 2e langue & 1,55 & 2e langue & 0,103 \\
\hline Langue étrangère & 1,3 & Langue de la science & 0,092 \\
\hline Langue du colonisateur & 0,61 & Langue de travail & 0,091 \\
\hline Langue indispensable & 0,51 & Langue de réussite & 0,091 \\
\hline Langue de travail & 0,4 & Langue de modernité & 0,09 \\
\hline Langue des études & 0,34 & Langue d'ouverture et de mobilité & 0,089 \\
\hline Langue d'ouverture et de mobilité & 0,16 & Langue des études & 0,087 \\
\hline Langue de modernité & $-0,04$ & $\begin{array}{l}\text { Langue de la communication quoti- } \\
\text { dienne }\end{array}$ & 0,084 \\
\hline Belle langue & $-0,2$ & Langue étrangère & 0,083 \\
\hline Langue de la science & $-0,42$ & Langue indispensable & 0,083 \\
\hline Langue de la réussite & $-0,55$ & Langue de prestige & 0,081 \\
\hline Langue de prestige & $-0,71$ & Langue difficile & 0,079 \\
\hline Langue difficile & $-0,89$ & Belle langue & 0,077 \\
\hline $\begin{array}{l}\text { Langue de la communication quoti- } \\
\text { dienne }\end{array}$ & $-0,95$ & Langue des mécréants & 0,071 \\
\hline Langue des mécréants & $-1,12$ & Langue du colonisateur & 0,059 \\
\hline
\end{tabular}

\section{Graphe de la représentation : Français}

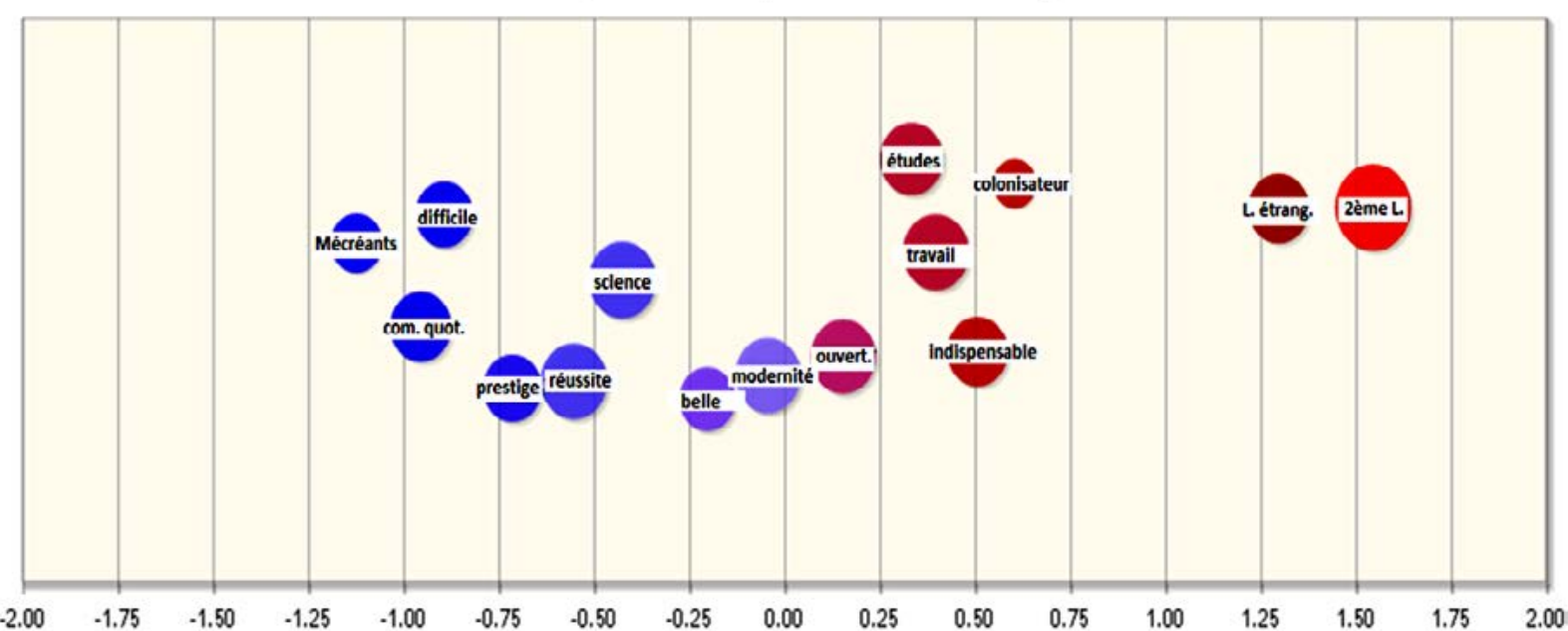

Graphe 7 : Graphe de la représentation sociale du français : adhésion - consensus 
Les items 6 ( $2^{\mathrm{e}}$ langue) et 1 (langue étrangère) se distinguent par une forte adhésion. L'indice de consensus est également très élevé pour le premier et significatif pour le second. De plus, ils représentent deux cognèmes fortement associés dans la représentation (le résultat du calcul de la distance est significatif). Ce qui les place dans le noyau de la représentation sociale (v. schéma en couronnes de la représentation).

A l'opposé, l'item 2 (langue des mécréants) occupe la zone de périphérie maximum de la représentation, avec le plus faible score d'adhésion, et un indice de consensus qui reste tout de même significatif.

L'item 11 (langue du colonisateur) apparait au troisième rang de l'adhésion, mais avec un score qui se détache nettement des deux premiers rangs. Cependant, le traitement dispersé dont il fait l'objet, qui se traduit par un indice de consensus très faible, va placer cet item dans la zone de périphérie incertaine.

L'ensemble des items restants, qui correspond à des images généralement positives du français, n'est pas marqué ; il occupe la couronne centrale de la représentation. Toutefois, nous pouvons distinguer au niveau de l'adhésion un groupe d'items valués positivement (langue indispensable, langue de travail, langue des études, langue d'ouverture et de mobilité) avec des degrés de consensus significatifs, et un autre groupe qui enregistre des scores d’adhésion négatifs.

\section{Schéma en couronnes de la structure de la représentation sociale du français}

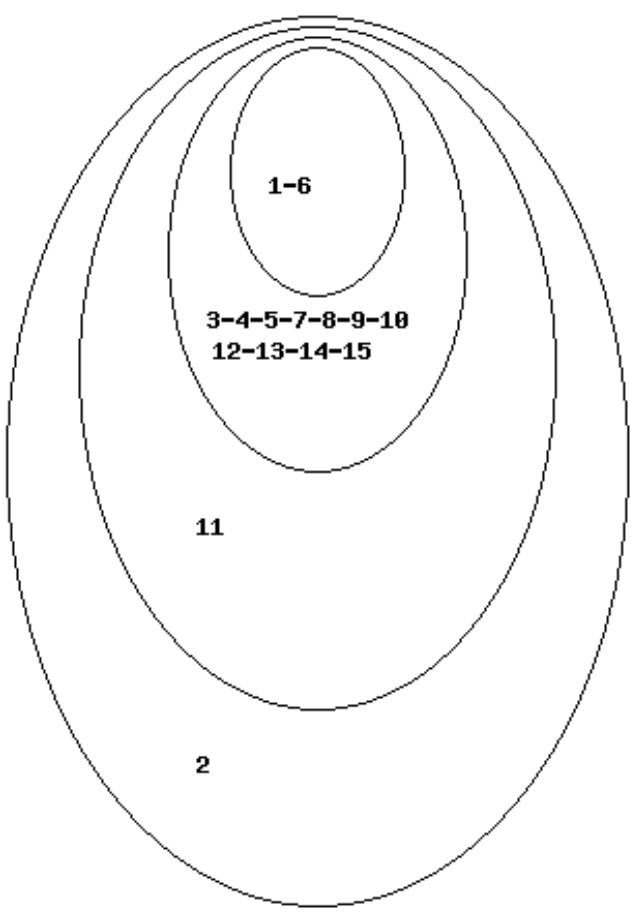

\begin{tabular}{|c|c|}
\hline Item $\mathbf{1}$ & Langue étrangère \\
\hline Item $\mathbf{2}$ & Langue de mécréants \\
\hline Item $\mathbf{3}$ & $\begin{array}{c}\text { Langue des études } \\
\text { Langue de la } \\
\text { communication } \\
\text { quotidienne }\end{array}$ \\
\hline Item $\mathbf{4}$ & Langue de prestige \\
\hline Item $\mathbf{5}$ & 2 $^{\text { langue en Algérie }}$ \\
\hline Item $\mathbf{6}$ & Ouverture mobilité \\
\hline Item $\mathbf{7}$ & Langue de la modernité \\
\hline Item $\mathbf{8}$ & Langue difficile \\
\hline Item $\mathbf{9}$ & Langue de la science \\
\hline Item $\mathbf{1 0}$ & Langue du colonisateur \\
\hline Item $\mathbf{1 1}$ & Langue indispensable \\
\hline Item $\mathbf{1 2}$ & Langue de travail \\
\hline Item $\mathbf{1 3}$ & Langue de réussite \\
\hline Item $\mathbf{1 4}$ & Belle langue \\
\hline Item $\mathbf{1 5}$ & \\
\hline
\end{tabular}

Graphe 8: Schéma en couronnes de la représentation sociale du français 


\subsubsection{Commentaire}

La représentation sociale du français est dominé par les deux cognèmes « 2 e langue » et « langue étrangère » qui ont enregistré le plus fort degré d'adhésion, qui sont également fortement associé (distance minimale) et que l'on retrouve finalement dans la zone de centralité maximale du schéma en couronne.

Ces deux images que l'on associe au français (à la fois « $2^{\mathrm{e}}$ langue » et « langue étrangère ») peuvent paraitre contradictoires. En tout cas, cette association semble bien refléter le statut ambigu qu'a le français en Algérie : officiellement langue étrangère mais fortement présent dans de nombreuses sphères sociales, longtemps stigmatisé dans le discours du pouvoir mais indispensable. Ces deux cognèmes qui émergent semblent donc traduire cette ambivalence, ce rapport d’attirance-répulsion.

C'est toutefois le cognème « $2^{\mathrm{e}}$ langue » qui est en tête, avec à la fois le plus fort degré d'adhésion et de consensus. Ce qui correspond bien à la réalité du terrain où le statut du français est plus proche de celui de «langue seconde $»^{17}$.

À l'autre extrémité, celle du rejet, nous avons le cognème «langue des mécréants », qui a le plus faible degré d'adhésion et que l'on retrouve dans la zone de périphérie marginale.

Le cognème « langue du colonisateur » enregistre un bon score d'adhésion mais fait l'objet du plus faible degré de consensus et n'est pas associé significativement à d'autres cognèmes. Voilà pourquoi ce cognème se retrouve dans la zone de périphérie incertaine.

Le rejet des cognèmes « langues des mécréants » et « langue du colonisateur » vers les zones périphériques de la représentation sociale, semble traduire une évolution de la représentation du français en Algérie. En effet, ces images, plus fortement présentes au lendemain de l'indépendance, sont aujourd'hui repoussées pour céder la place à une représentation qui met au premier plan l'intérêt fonctionnelle du français.

C'est ainsi que dans la couronne centrale viennent se concentrer essentiellement des cognèmes qui font ressortir l'intérêt pragmatique du français ainsi que son image positive (langue de travail, langue indispensable, langue d'ouverture et de mobilité, langue de la science, etc.). Certains sont même fortement associés («langue de la science » avec «langue de la réussite »; « langue d'ouverture et de mobilité » avec «langue de modernité »).

17. Selon Louise Dabène (1994 : 107), une langue seconde est « une langue étrangère qui est dotée, par suite de circonstances historiques particulières et de la situation sociolinguistique qui en est corollaire, d'un statut privilégié, et qui participe, comme langue d'enseignement, au développement psychologique et cognitif de l'enfant, puis, de façon privilégiée, aux capacités informatives de l'adulte (médias, documentation professionnelle et administrative...) ». 
Ces résultats s'inscrivent en cohérence avec la configuration sociolinguistique de l'Algérie actuelle. En effet, à côté des faiblesses voire de l'échec de l'arabisation, le français continue d'occuper une place importante voire prépondérante dans de nombreuses sphères sociales ou secteurs d'activité. En conséquence, cette langue, souvent indispensable, est perçue comme une langue de réussite et d’ascension sociale, un élément de la « distinction» (Bourdieu, 1979).

Enfin, le cognème 7 (langue d'ouverture et de mobilité) traduit le rôle que joue la langue française dans la circulation migratoire, et en particulier la mobilité académique des étudiants algériens.

\section{Conclusion}

L'enquête que nous avons menée, grâce à une approche structurale de la représentation sociale (Maurer, 2013), a permis de jeter un nouveau regard sur les représentations sociales des différentes langues en contact en Algérie. L'analyse a mis en exergue les multiples composantes de ces représentations et a mis au jour, pour notre population, celles qui émergent avec un fort degré d'adhésion et celles qui sont moins consensuelles. Ainsi, les résultats de cette étude ont permis d'apprécier la prégnance des images que nos enquêtés associent aux différentes langues en présence en Algérie.

L'arabe standard reste fortement associé, dans l'esprit des enquêtés, à la religion et l'identité nationale; ce qui entre en cohérence avec le discours idéologique qui a longtemps prévalu. Les valeurs emblématiques qui lui sont associées montrent les rapports positifs à cette langue que beaucoup considèrent comme « langue maternelle » même si elle absente de l'usage quotidien des locuteurs. L'arabe dialectal, quant à lui, se voit associer des images plutôt positives, en lien avec son utilité fonctionnelle et sa vitalité (les items « langue des analphabètes », « langue inutile » et « non langue » se situant du côté du rejet).

Par ailleurs, la réhabilitation du berbère comme évènement politique et sa promotion sur le terrain (dans les domaines de l'éducation et des médias notamment) semblent avoir été assez bien suivies au niveau de la représentation sociale. En effet, nous avons observé l'adhésion à des cognèmes comme « langue du patrimoine », « langues des origines », « symbole de l'identité culturelle »; et une tendance à rejeter les cognèmes « langue inutile », « non langue » et « langue de conflit ».

Enfin, ce sont des images positives qui entourent le français. En effet, l'image du français comme langue du colonisateur, autrefois très prégnante, semble bien prendre du recul pour céder la place à des cognèmes traduisant l'intérêt fonctionnel de cette langue qui occupe aujourd'hui une place de choix sur le marché linguistique.

Finalement, au-delà des idées reçues voire dictées par l'idéologie, l'approche que nous avons utilisée pour cette enquête nous a permis de faire des constats sur les représentations sociales mais aussi des hypothèses sur une dynamique des représentations qui est en cours et en lien avec la dynamique sociolinguistique et l'action glottopolitique. 


\section{Bibliographie}

Abbès-Kara, Attika-Yasmine (2011), "La variation dans le contexte algérien : enjeux linguistique, socioculturel, et didactique », Cahiers de sociolinguistique, n 15 (Approches de la pluralité sociolinguistique : vers quelles convergences de pratiques de recherche et d'éducation, sous la dir. d'Isabelle Pierozak, Thiery Bulot et Philippe Blanchet), p. 77-86.

Ait-Hamou Ali, Rabiha (2004), La place du français dans le discours épilinguistique de lycéen tiziouzéens : approche praxématique, thèse de doctorat, Tizi-Ouzou, Université Mouloud Mammeri.

Amrane, Katia Myriam (2010), "Pour une analyse de la représentation de l'identité ou des identités algériennes en contexte discursif », dans Philippe Blanchet, Malika Kebbas et Attika-Yasmine Abbès-Kara (dir.), Influences et enjeux des contextes plurilingues sur les textes et les discours, Limoges, Lambert-Lucas, p. 37-72.

Arezki, Abdenour (2010), "La planification linguistique en Algérie ou l'effet de boomerang sur les représentations sociolinguistiques », Le français en Afrique, n²5, p. 165-171.

Bektache, Mourad (2014), "Minoration et dénomination des parlers berbères », dans Romain Colonna (dir.), Les locuteurs et les langues: pouvoir, non-pouvoir et contre-pouvoir, Limoges, Lambert-Lucas, p. 212-232.

Benrabah, Mohamed (1993), "L’arabe algérien véhicule de la modernité », Cahiers de linguistique sociale, n²2 (Minoration linguistique au Maghreb, sous la dir. de Fouad Laroussi), p. 33-43.

Benrabah, Mohamed (1999), Langue et pouvoir en Algérie : histoire d’un traumatisme, Paris, Séguier.

Blanchet, Philippe, Louis-Jean Calvet et Didier de Robillard (dir.) (2007), Un siècle après le cours de Saussure : la linguistique en question, numéro thématique des Carnets d'Ateliers de Sociolinguistique, $\mathrm{n}^{\circ} 1$.

Boucherit, Aziza (1991), "Convergence et résistance des hommes et des langues », International journal of the sociology of language, n 87, p. 55-69.

Boudebia-Baala, Afaf (2012), L'impact du contexte sociolinguistique et scolaire sur l'enseignement/ apprentissage du français dans le Souf à travers l'analyse des représentations comme outil de description, thèse de doctorat, Besançon, Université de Franche-Comté.

Bourdieu, Pierre (1979), La distinction : critique sociale du jugement, Paris, Éditions de Minuit.

Breton, Roland (1999), «Solidité, généralisation et limites du modèle “jacobin" de politique linguistique face à une nouvelle Europe », dans Philippe Blanchet, Roland Breton et Harold Schiffman (dir.), Les langues régionales de France: un état des lieux à la veille du XXIe siècle/The Regional Languages of France: an inventory on the eve of the XXI century. Actes du colloque organisé à I'Université de Pennsylvanie, Philadelphie, 4-6 octobre 1996, Louvain, Peeters, coll. "Bibliothèque des Cahiers de Linguistique de Louvain, 102 », p. 81-94. 
Bourhis, Richard Y. et Rodrigue Landry (2002), « La loi 101 et l'aménagement du paysage linguistique au Québec », Revue d'aménagement linguistique, hors série (L'aménagement linguistique au Québec: 25 ans d'application de la Charte de la langue française, sous la dir. de Pierrre Bouchard et Richard Bourhis), p. 107-132.

Calvet, Louis-Jean (1974), Linguistique et colonialisme : petit traité de glottophagie, Paris, Payot.

Calvet, Louis-Jean et Alain Calvet (2013), Les confettis de Babel : diversité linguistique et politique des langues, Paris, Éditions Écritures.

Chachou, Ibtissem (2013a), La situation sociolinguistique d’Algérie : pratiques plurilingues et variétés à l'oeuvre, Paris, L'Harmattan.

Chachou, Ibtissem (2013b), "Le hiatus "pratiques vs représentations" en sociolinguistique algérienne: vers une relativisation du constat », dans Violaine Bigot, Aude Bretegnier et Marie-Thérèse Vasseur (dir.), Vers le plurilinguisme? Vingt ans après, Paris, Éditions des archives contemporaines, p. 195-201.

Chériguen, Foudil (dir.) (2007), Les enjeux de la nomination des langues dans l'Algérie contemporaine, Paris, L'Harmattan.

Colonna, Romain (2013), Les paradoxes de la domination linguistique : la diglossie en question, Paris, L'Harmattan.

Dabène, Louise (1994), Repères sociolinguistiques pour l'enseignement des langues, Paris, Hachette.

Elimam, Abdou (2004), Langues maternelles et citoyenneté en Algérie, Oran, Éditions Dar El Gharb.

Gasquet-Cyrus, Médéric et Cécile Petitjean (2009), Le poids des langues : dynamiques, représentations, contacts, conflits, Paris, L'Harmattan.

Grine, Nadia (2009), Les représentations linguistiques et leur incidence sur la réussite ou l'échec d'une politique linguistique, thèse de doctorat, Mostaganem, Université Abdelhamid Benbadis.

Grandguillaume, Gilbert (1983), Arabisation et politiques linguistiques au Maghreb, Paris, Maisonneuve et Larose.

Grandguillaume, Gilbert (2010), «L'Algérie pays francophone ? », dans Katia Malausséna et Gérard Sznicer (dir.), Traversées francophones, Genève, Éditions Suzanne Hurter, p. 102-114.

Houdebine-Gravaud, Anne-Marie (2002), «L'imaginaire linguistique : un niveau d'analyse et point de vue théorique », dans Anne-Marie Houdebine-Gravaud (dir.), L'imaginaire linguistique, Paris, L'Harmattan, p. 9-21.

Longatte, Annie (1999), «Pratiques langagières de la deuxième génération de l'immigration maghrébine en France », dans Ernstpeter Ruhe (dir.), Die Kinder der Immigration : les enfants de l'immigration, Würzburg, Königshausen \& Neumann, p. 65-82. 
Maurer, Bruno (2011), « Méthodologie d'enquête pour une représentation graphique des composants de la représentation sociale d'une langue », dans Philippe Blanchet et Patrick Chardenet (dir.), Guide pour la recherche en didactique des langues et des cultures: approches contextualisées, Paris, Éditions des archives contemporaines, p. 179-192.

Maurer, Bruno (2013), Représentations des langues en situation multilingue, Paris, Éditions des archives contemporaines.

Morsly, Dalila (1988), Le français dans la réalité algérienne, thèse de doctorat d'État, Paris, Université Paris V Sorbonne.

Morsly, Dalila (1990), «Attitudes et représentations linguistiques », Linguistique, vol. 26, nº 2 (Linguistique et «facteurs externes»), p. 77-86.

Sini, Chérif (dir.) (2013), Les langues dans l'espace familial algérien, Oran, Éditions du CRASC.

Quéffelec, Ambroise, Yacine Derradji, Valéry Debov, Dalila Smaali-Dekdouk et Yasmina CherradBenchefra (dir.) (2002), Le français en Algérie: lexique et dynamique des langues, Bruxelles, Deboeck et Larcier.

Taleb-Ibrahimi, Khaoula (1997), Les Algériens et leur(s) langue(s), Alger, Dar El Hikma.

Taleb-Ibrahimi, Khaoula (2004), "L'Algérie : coexistence et concurrence des langues », L'Année du Maghreb, nº 1, p. 207-218.

Trimaille, Cyril et Marinette Matthey (2013), «Catégorisations », dans Jacky Simonin et Sylvie Wharton (dir.), Sociolinguistique du contact : dictionnaire des termes et des concepts, Lyon, ENS Éditions, p. 95-122.

Sebaa, Rabeh (2002), L’Algérie et la langue française : l'altérité partagée, Oran, Éditions Dar El Gharb.

Zenati, Jamel (2004), «L'Algérie à l'épreuve de ses langues et de ses identités : histoire d'un échec répété », Mots, $n^{\circ} 74$ (Langue(s) et nationalisme(s), sous la dir. d'Henri Boyer), p. 137-145. 


\section{Annexes}

\section{Questionnaire de la pré-enquête}

\section{Questionnaire (pré-enquête)}

Ce questionnaire est destiné à une recherche universitaire sur les représentations sociales des langues en Algérie. Les réponses recueillies seront anonymes (inutile d’écrire votre nom).

Merci de votre collaboration.

Consigne : Que représente pour vous chacune des langues suivantes : l'arabe standard, l'arabe dialectal, le berbère, le français? Vous pouvez répondre en français ou en arabe.

Pour moi, l'arabe standard est:

Pour moi, l'arabe dialectal est:

Pour moi, le berbère est:

Pour moi, le français est: 
Consigne : Que représente pour toi la langue arabe standard? Classe les propositions suivantes de 1 à 15.

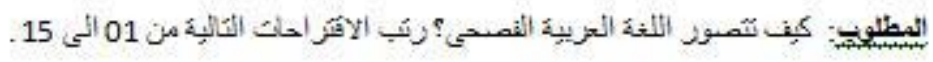

de 1

Pour moi, c'est vraiment ça.

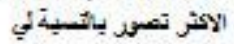

$>$ à 15

Pour moi, ce n’est pas du tout ça.

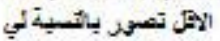
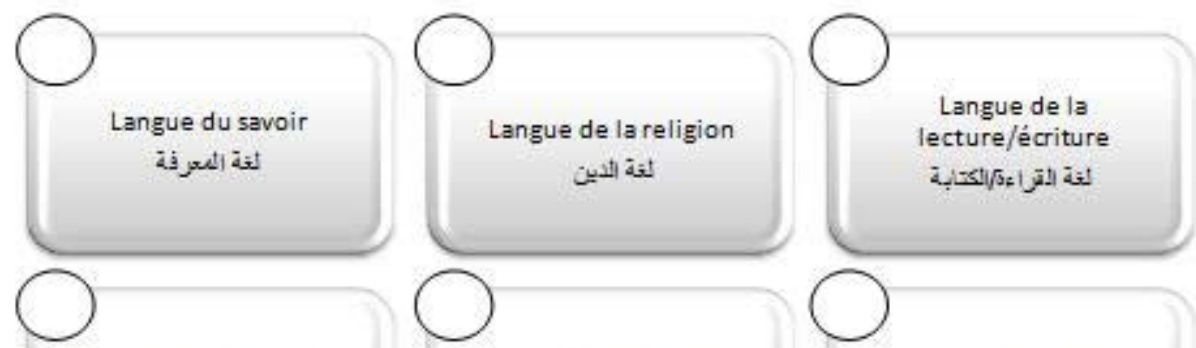

langue de l'administration

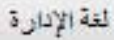

Langue de l'école

كلية المبربسة
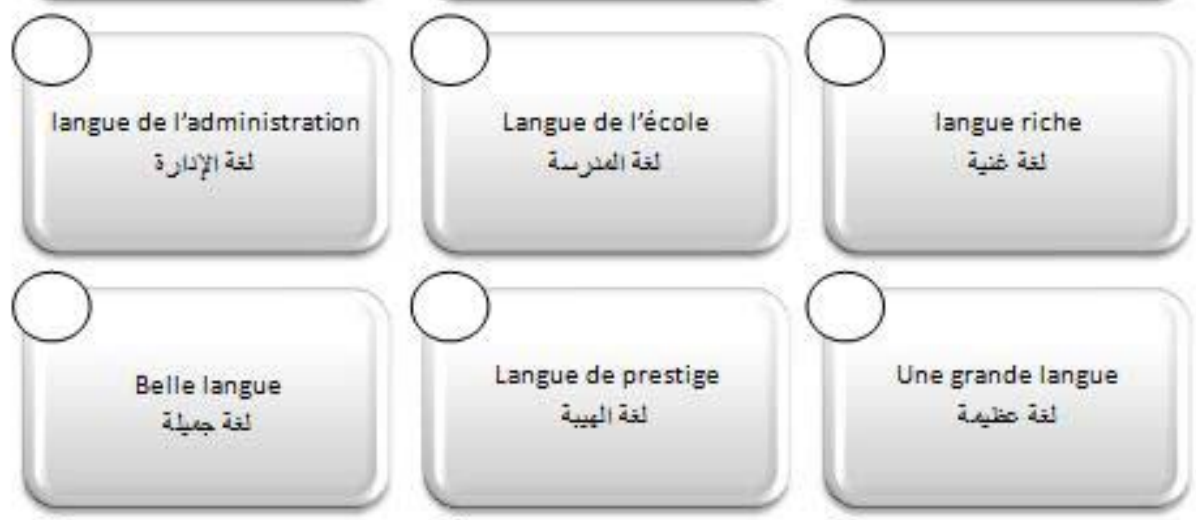

$\bigcirc$

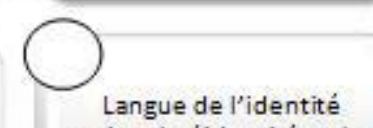
nationale /identité arabe

لئة ألهوية الوطنيه الثهوية العربية

الثة عمبة لية
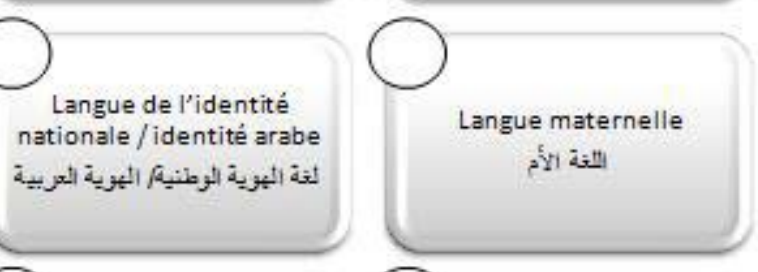

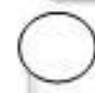
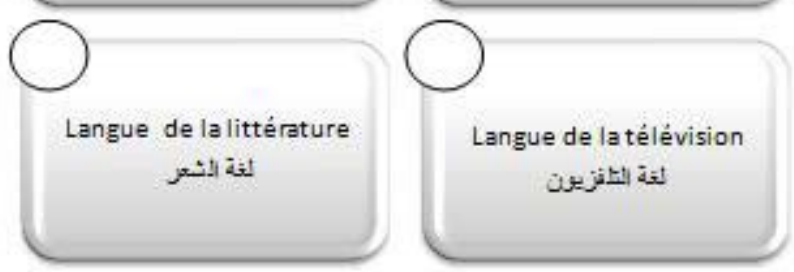

Langue des origines

الثية الأصول (الأجداند)

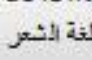

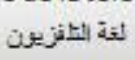


Consigne : Que représente pour toi l'arabe dialectal ? Classe les propositions suivantes de 1 à 15 .

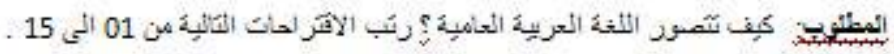

de 1

Pour moi, c'est vraiment ça.

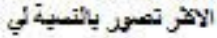

Pour moi, cen'est pas du tout ca

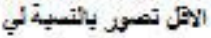

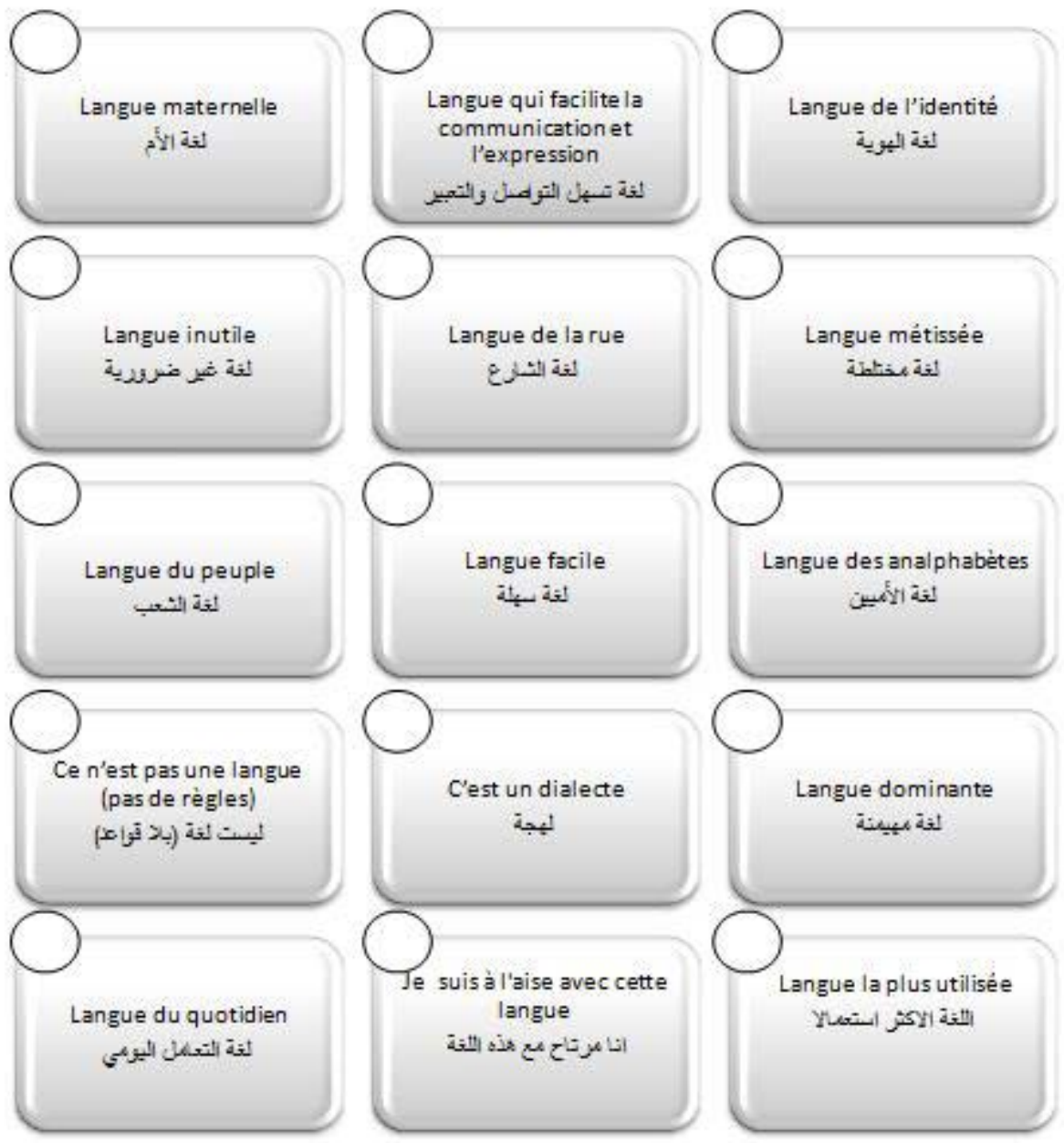


Consigne : Que représente pour toi le berbère ? Classe les propositions suivantes de 1 à 15 .

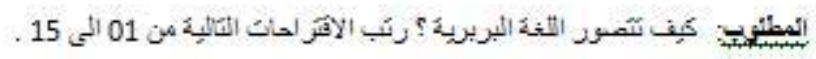
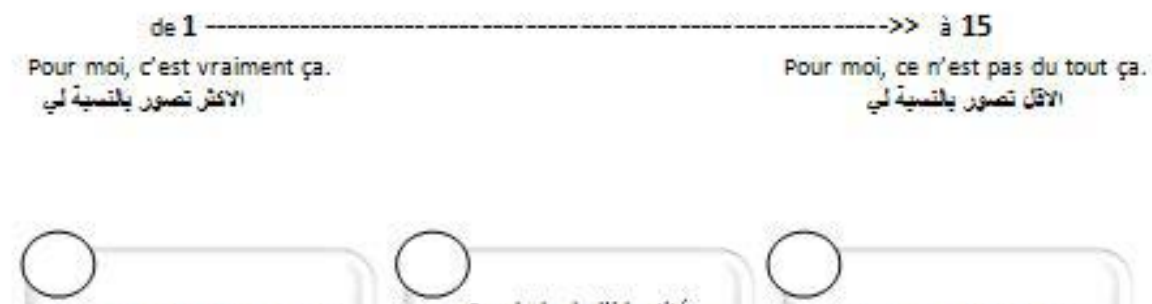

Langue des origines, des ancêtres

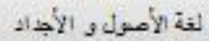

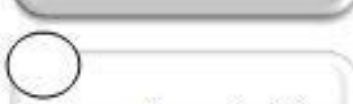

Langue d'une minorité, langue régionale

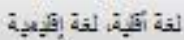

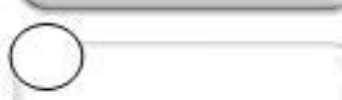

Langue secondaire as

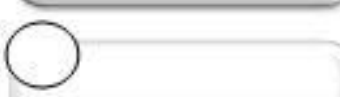

Langue des Maghrébins لفة هيلريية

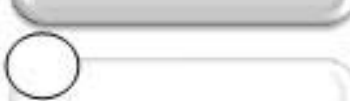

Langue de conflit interethnique et intra-ethnique

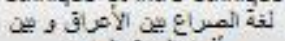

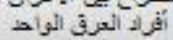

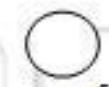

symbole de Pidentité culturelle

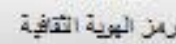

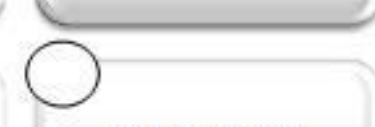

Langue étrangère لزة نجنية
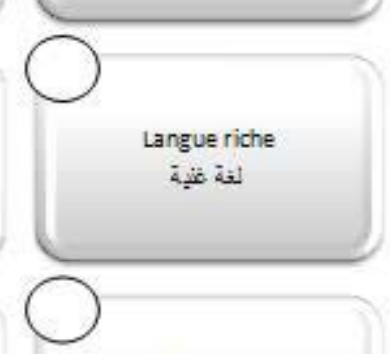

Belle langue لفة جيلة

$\bigcirc$

Ce n'est pas une langue, c'est une sous-langue

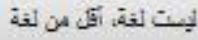
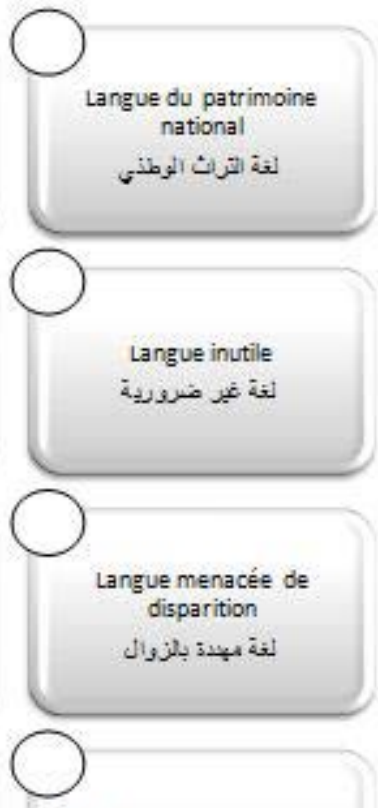

Dialecte, Langue de loralité

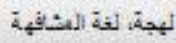
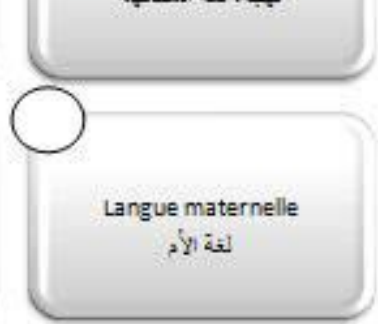
Consigne : Que représente pour toi le francais ? Classe les propositions suivantes de 1 à 15.

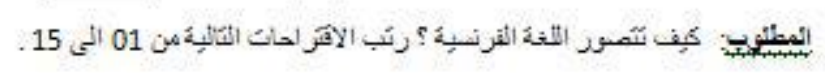

de 1 Pour moi, c'est vraiment ça.

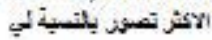

\section{à 15}

Pour moi, ce n'est pas du tout ça. الاقد تصنوز بلتصبت لي
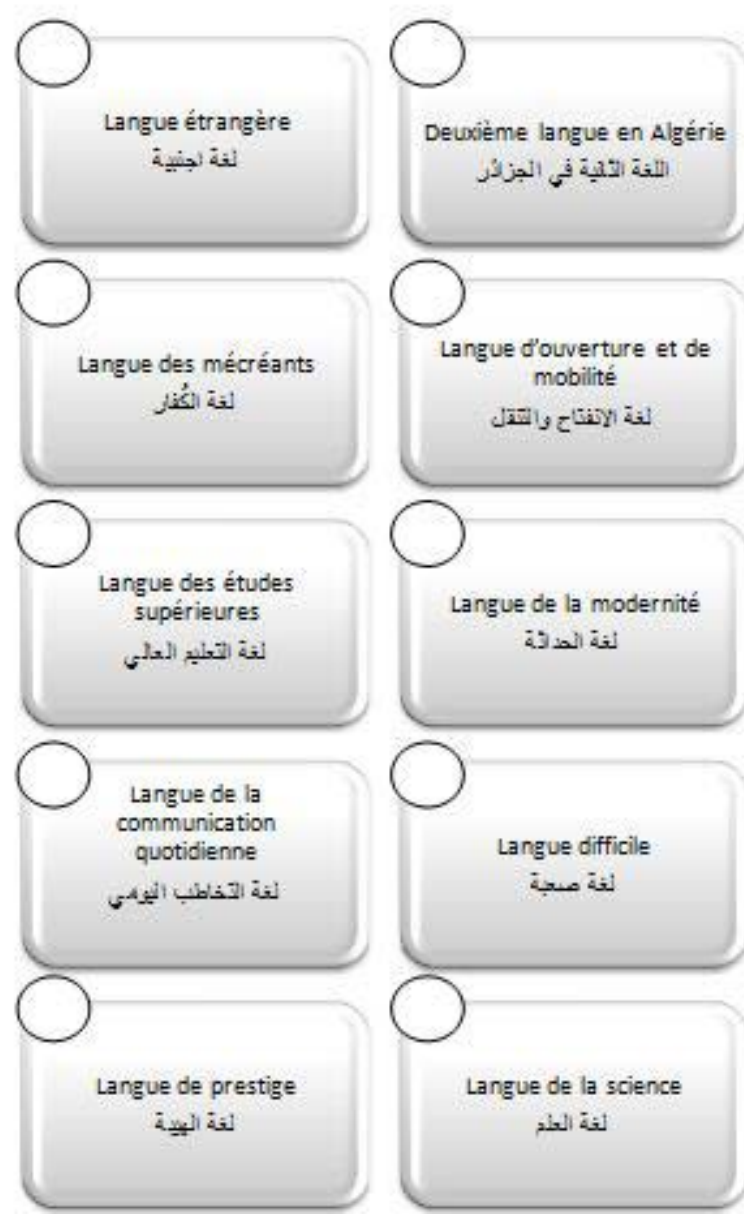

Langue d'ouverture et de mobilité

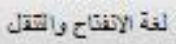
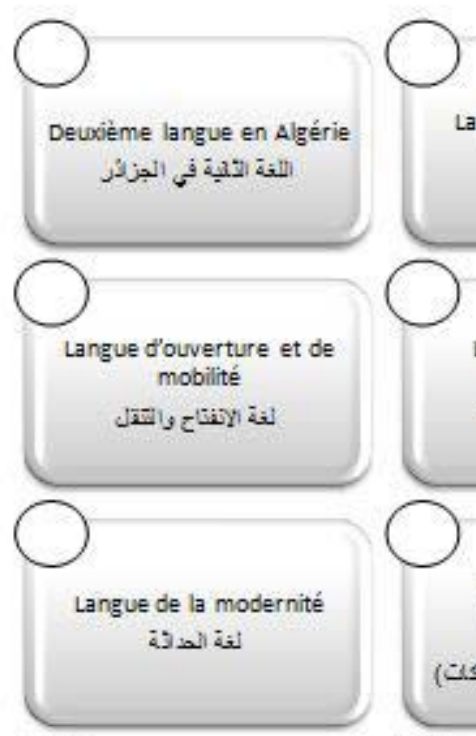

Langue du colonisateur

$$
\text { Jivill }
$$
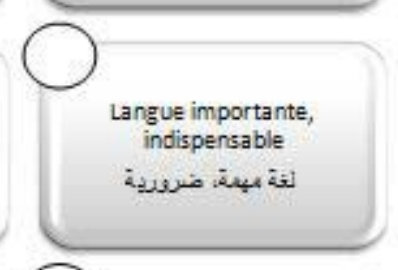

(

Langue de travail

[administrations,

entreprises...]

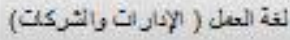

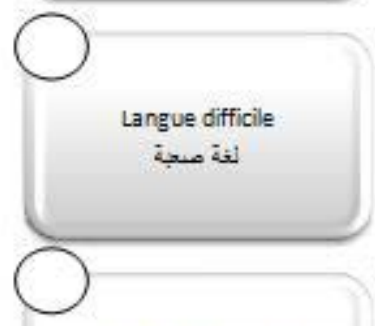

Langue de la science لغة الطذد

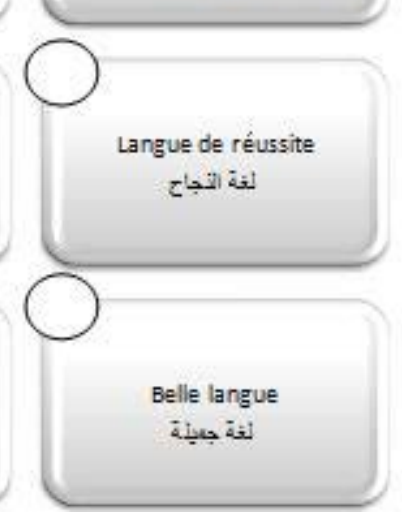

